Supporting Information for:

\title{
Synthesis of a new trans- $A_{2} B_{2}$ phthalocyanine motif as a building block for rod-like phthalocyanine polymers
}

\author{
W. Justin Youngblood
}

\section{Table of Contents}

1. General Experimental Section.

2. Photochemistry of Benzimidazoporphyrazines

3. Sample ${ }^{1} \mathrm{H}$ NMR of $\mathrm{d}_{8}$-THF (Cambridge Isotopes).

4. Spectral data for compounds 3, 5a, 5d, 6b, Fb-7, Mg-7, Zn-7, and Zn-10.

5. Spectral data for compounds Fb-11, Zn-11, Zn-12, Fb-13, and Zn-13ab. [second SI file]

6. Spectral data for compounds Zn-13a, Zn-13b, Zn-17a, Zn-17b. [third SI file]
Page(s)

S1

S2-S9

S10

S11-S50

S51-S82

S83-S110

1. General. ${ }^{1} \mathrm{H}(400 \mathrm{MHz})$ and ${ }^{13} \mathrm{C}(75 \mathrm{MHz})$ NMR spectra were obtained in $\mathrm{CDCl}_{3}$ except where noted otherwise. Silica gel (Scientific Adsorbents, $40 \mu \mathrm{m}$ average particle size) was used for column chromatography. Anhydrous $\mathrm{CH}_{2} \mathrm{Cl}_{2}$ was purchased from Aldrich. Aldehydes $\mathbf{4} \mathbf{b}$ and $\mathbf{4} \mathbf{c}$ and all other chemicals were purchased as reagent grade and were used as received. Benzimidazoles $\mathbf{5 b}$ and $\mathbf{5} \mathbf{c}$ were not isolated but were carried forward to the corresponding products $\mathbf{6 b}$ and $\mathbf{6 c}$. Photochemical data were measured using THF as solvent. Molar absorption coefficients were measured by dissolving a known quantity of compound in $250 \mathrm{~mL}$ of THF in a volumetric flask and recording the absorption spectrum of the resulting solution. Fluorescence quantum yields were measured by comparison to tetratert-butylphthalocyanine, which was itself measured in both THF and $\mathrm{CHCl}_{3}$ set at $\Phi_{\mathrm{f}}=0.70$ in THF (by comparison to the reported $\Phi_{\mathrm{f}}$ of 0.77 for tetra-tert-butylphthalocyanine in $\mathrm{CHCl}_{3}$ with correction for the indices of refraction of the different samples). ${ }^{1 a}$ 
2. Photochemistry of Benzimidazoporphyrazines. The spectra of $A_{4}$ benzimidazoporphyrazines are typical of phthalocyanines, with broad B bands in the 300-400 nm region, and sharper Q bands providing strong absorbance in the red/near-IR portion of the spectrum. The absorption and emission spectra of compounds Fb-7 and Zn-7 (in THF) are shown in Figure S1.
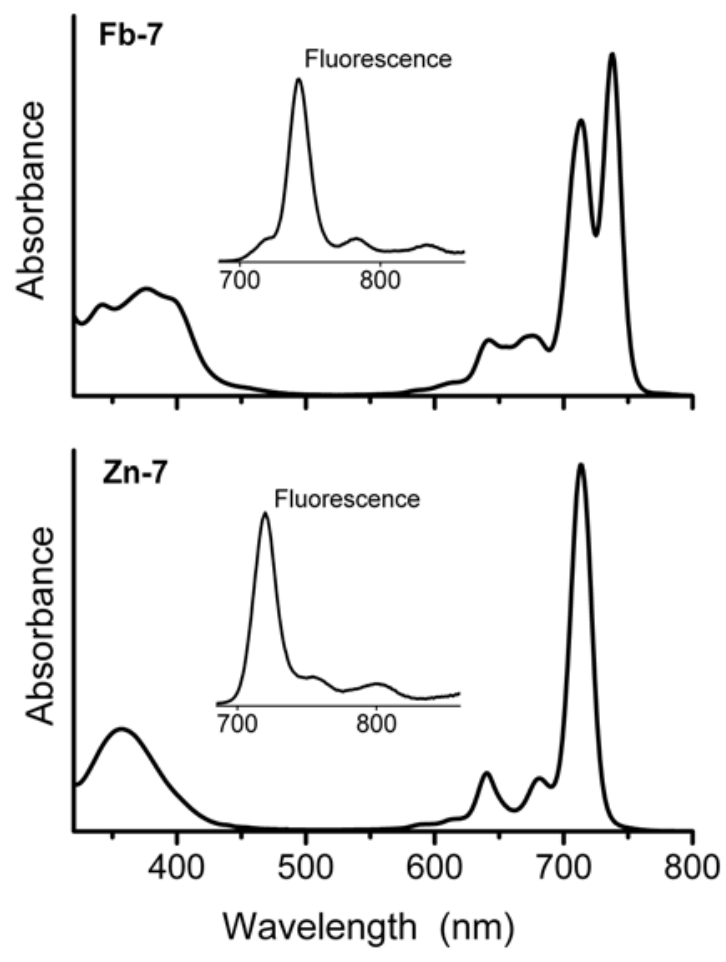

FIGURE S1. Representative absorption and emission spectra for tetrasubstituted free base and metallo-benzimidazoporphyrazines (in THF). A generic structure for the compounds is shown in Scheme 3.

The extinction coefficients are somewhat higher than the reported values for unsubstituted phthalocyanines and even the more soluble tetra-tert-butylphthalocyanines, ${ }^{1}$ but this may simply be an effect of the narrowness of the Q band maxima for the BzImPAs (Table S1). The spectral profiles of the magnesium-metallated tetrabenzimidazoporphyrazines are similar to the zinc-metallated species (see Table S1 and additional spectra in Supporting Information). The spectra for the phenyl-substituted series M-8 closely match those of the alkyl series $\mathbf{M - 7}$, but are red-shifted by 2-4 nm. Of all the 
benzimidazoporphyrazines in this study, only Fb-8 does not obey the Beer-Lambert law. For the others, no significant change is observed in the UV-Vis absorbance profile between solutions having maximal absorbance in the range 0.02-2.0 absorbance units. Fb-8 showed a 15\% decrease in the ratio of the B band to the Q band between solutions at 0.02 and 2.0 absorbance units, indicating a stronger propensity for aggregation of the species in solution.

TABLE S1. Photochemical data for benzimidazoporphyrazines (in THF).

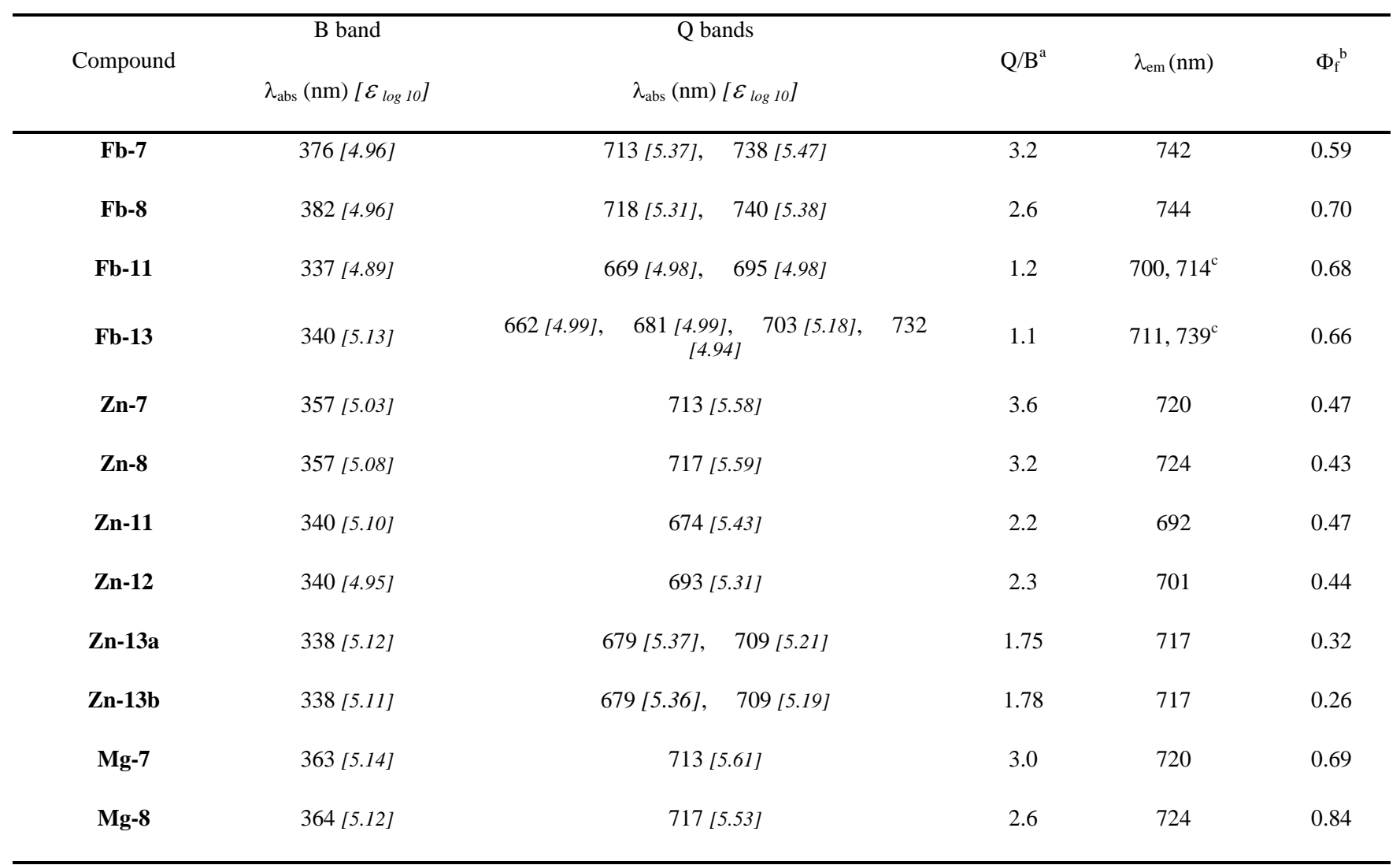

${ }^{\mathrm{a}}$ Ratios of intensities were calculated from absorbance data. Q value was chosen from the most intense Q band for a given compound.

${ }^{\mathrm{b}}$ The method for quantum yield determination is described in the General Experimental Section.

${ }^{\mathrm{c}}$ The fluorescence profile of the sample was dependent upon the wavelength of excitation. 
The $\mathrm{Q}$ bands of the $\mathrm{A}_{4}$ BzImPAs are red-shifted by 35-50 nm compared to the corresponding free base or metallophthalocyanines. ${ }^{1}$ The $\mathrm{Q}$ band maxima for various phthalocyanine species in THF are as follows: zinc phthalocyanine (ZnPc, 665 nm/15,038 cm $\left.{ }^{-1}\right){ }^{2}$ Zn-7 (713 nm/14,225 cm ${ }^{-1}$, Table S1), and zinc naphthalocyanine (ZnNc, $\left.756 \mathrm{~nm} / 13,228 \mathrm{~cm}^{-1}\right)^{2}$ The excited-state energy gap of $\mathbf{Z n - 7}$ is intermediary in this group, being $813 \mathrm{~cm}^{-1}$ less than that of ZnPc, and $997 \mathrm{~cm}^{-1}$ greater than that of ZnNc. The $\pi$-system of $\mathbf{Z n - 7}$ has the same number of electrons as ZnNc, but four fewer nuclei. This translates to four fewer molecular orbitals via the LCAO formalism, and explains why the BzImPAs are blue-shifted compared to naphthalocyanines. Another distinction of the imidazole annulation is that the moderate red-shift of the phthalocyanine electronic transitions comes without any sacrifice in the fluorescence quantum yield. Whereas the $\Phi_{\mathrm{f}}$ of $\mathbf{Z n N c}(0.13)^{2}$ is reduced from that of $\mathbf{Z n P c}(0.32),{ }^{2}$ the $\Phi_{\mathrm{f}}$ of $\mathbf{Z n - 7}$ is higher (0.47).

The lower symmetry of free base macrocycles Fb-11 $\left(\mathrm{A}_{3} \mathrm{~B}\right)$ and $\mathbf{F b}-\mathbf{1 3}$ (trans- $\mathrm{A}_{2} \mathrm{~B}_{2}$ ) results in very different behavior from the $\mathrm{A}_{4}$ BzImPAs. Figure S2 shows the absorption profiles of the $\mathrm{A}_{3} \mathrm{~B}$ and trans$\mathrm{A}_{2} \mathrm{~B}_{2}$ compounds. The $\mathrm{Q}$ band maxima are more complex, and the $\mathrm{Q}$ band intensity is dropped to roughly the same as the $\mathrm{B}$ band. The altered $\mathrm{Q} / \mathrm{B}$ ratio is not the result of aggregation, as no difference was observed in the absorption profile between solutions having maximal absorptions of 0.02 and 2.0 absorbance units. 

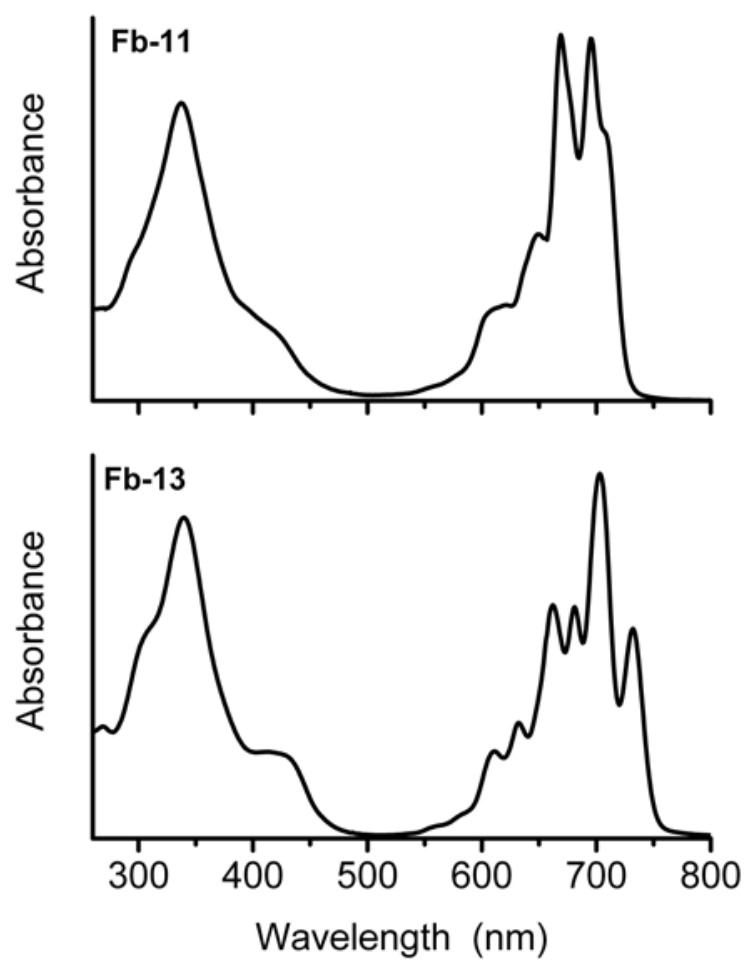

FIGURE S2. Absorption spectra for low symmetry free base BzImPAs (in THF). Structures of the compounds are shown in Schemes 5 and 6.

The standard free base phthalocyanine system has two distinct transition dipole moments, along the two N-N axes. The two transition dipole moments, normally labeled as $\mathrm{Q}_{\mathrm{x}}$ and $\mathrm{Q}_{\mathrm{y}}$, for the pyrrole-like and pyridine-like axes respectively, give rise to the two maxima normally observed in the red/near-IR region of the electromagnetic spectrum. Fb-7 (Figure S1) and Fb-8 exhibit this spectral pattern. For the free base $\mathrm{A}_{3} \mathrm{~B}$ (Fb-11) and trans- $\mathrm{A}_{2} \mathrm{~B}_{2}(\mathbf{F b}-\mathbf{1 3})$ benzimidazoporphyrazines, the difference in annulation along the N-N axes also affects the multiplicity of transition dipoles. The $\mathrm{Q}_{\mathrm{x}}$ and $\mathrm{Q}_{\mathrm{y}}$ dipoles give rise to different electronic transitions depending on their placement on the short or elongated axis of the macrocycle. The tautomers of each free base compound are therefore photochemically distinct from each other. Each spectrum in Figure S2 is an overlay of the absorption profiles of two tautomeric chromophores. 
This hypothesis of photochemically distinct tautomers is supported by the fluorescence behavior of Fb-11 and Fb-13: each compound appears to have two strong fluorescence maxima instead of the standard single fluorescence maximum for a free base phthalocyanine (Figure S3). Additionally, the intensities of the two maxima change independently of each other upon altering the wavelength of excitation. The two tautomers of a given low symmetry free base macrocycle may each be responsible for one emission maximum and accompanying vibronic bands. If these varying emission profiles were the result of individual photochemical species, then the compounds would be in violation of Kasha's rule: excited species must always pass through the same lowest singlet excited state before returning to the ground state by whatever means (typically internal conversion, triplet crossover, or fluorescence). Species that obey Kasha's rule exhibit a single fluorescence spectrum regardless of the wavelength of excitation. A simple explanation of the complex absorption and fluorescence profiles of $\mathbf{F b}-\mathbf{1 1}$ and $\mathbf{F b}$ 13 is that they do not result from singular sets of electronic transitions, but rather are each an overlay of the absorption/fluorescence bands due to pairs of photochemically distinct tautomers.

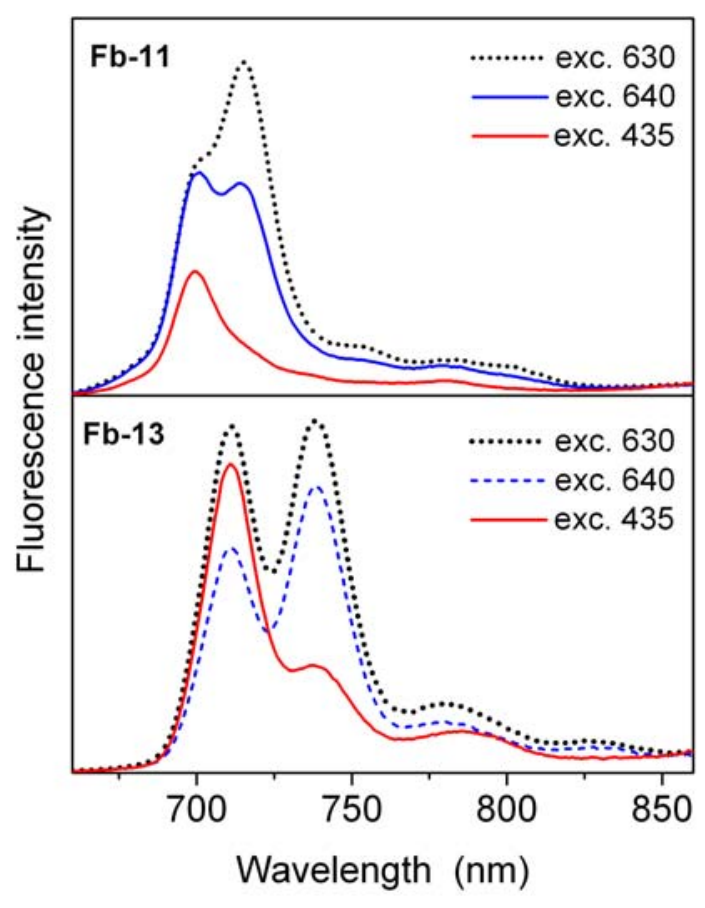

FIGURE S3. Emission spectra of low-symmetry free base BzImPAs (in THF). Structures of the compounds are shown in Schemes 5 and 6. 
Metallation of the phthalocyanine scaffold removes the effect of tautomerism on the photochemical behavior of the resulting chromophores. This clarifies the contribution of the differential annulation in the photochemical behavior of Zn-11 and Zn-13. Whereas the simple unsubstituted metallophthalocyanine Zn-10 has a single Q-band absorption maximum, Zn-13 (trans- $\mathrm{A}_{2} \mathrm{~B}_{2}$ ) has a double Q band (Figure S4). Zn-11 has a tall shoulder on the longer wavelength side of the band, and may have two distinct transition dipole moments as in Zn-13, with the two dipole moments overlapping so extensively as to appear to be one band. The zinc chelates Zn-11 and Zn-13 each show a single emission maximum, in contrast to their free base analogs, and the fluorescence profiles of the zinc chelates do not change with varying the wavelength of excitation. $\mathbf{Z n - 1 2}$, the cis- $\mathrm{A}_{2} \mathrm{~B}_{2}$, also has a single emission maximum, as well as a single Q-band absorption maximum, but these were expected, as the two axes of Zn-12 are identical with respect to their extent of annulation (see additional spectra in Supporting Information).

The two regioisomers of $\mathbf{Z n - 1 3}$, of $\mathrm{C}_{2 \mathrm{~h}}$ and $\mathrm{C}_{2 \mathrm{v}}$ symmetry, show nearly identical spectra. The extinction coefficients for the absorption maxima of $\mathbf{Z n - 1 3 b}$ are minutely smaller than those of $\mathbf{Z n - 1 3 a}$, and the ratio of the intensity of the two Q bands to one another in $\mathbf{Z n - 1 3 a}$ is slightly lower (3\%) than the corresponding ratio of Q bands in Zn-13b (Table S1). The fluorescence quantum yields are slightly different, but both are still within the known range for zinc phthalocyanine-type compounds. These two diethynyl constructs are essentially equivalent and to each other as well as to the deprotected derivatives Zn-17a and Zn-17b. The double $Q$ band appearance found in these trans- $A_{2} B_{2}$ compounds is very similar to the absorption profile of a trans- $\mathrm{A}_{2} \mathrm{~B}_{2}$ zinc phthalocyanine presented by Kobayashi, et al., ${ }^{2}$ wherein one axis is phthalocyanine-like and the other axis is naphthalocyanine-like. However, that trans- $\mathrm{A}_{2} \mathrm{~B}_{2}$ compound also exhibited a complex emission profile unlike the zinc-BzIMPAs shown here. 

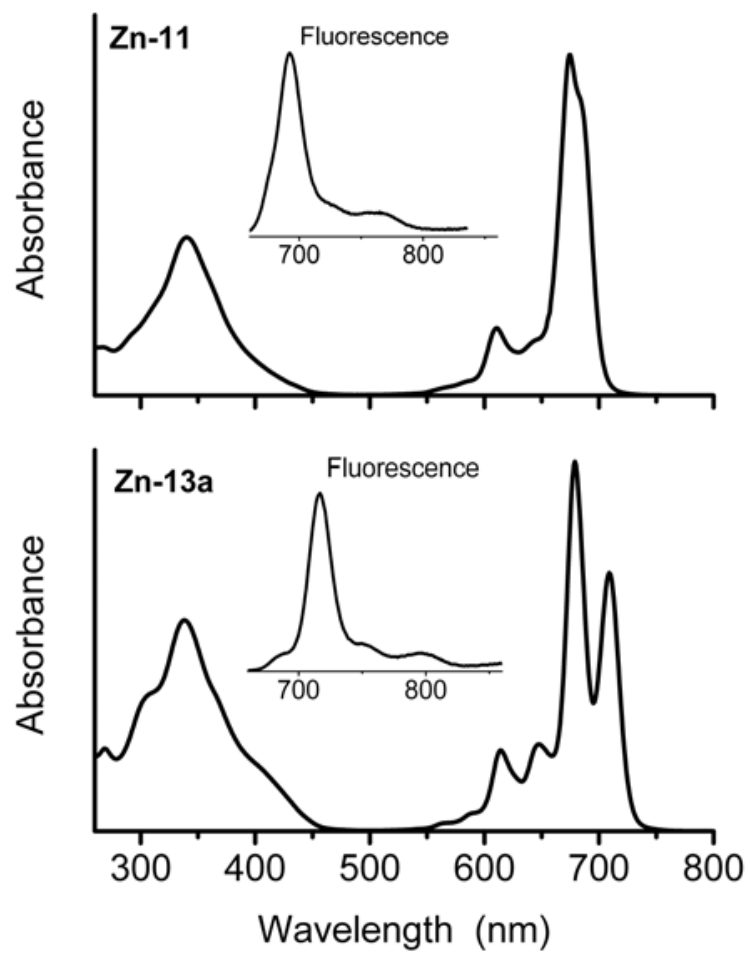

FIGURE S4. Absorption and emission spectra for low symmetry Zn-BzImPAs (in THF). Structures of the compounds are shown in Schemes 4 and 6.

\section{Conclusion}

The photochemical characteristics of some $A_{4}, A_{3} B$, and $A_{2} B_{2}$ benzimidazoporphyrazines have been detailed. Benzimidazoporphyrazines are essentially phthalocyanine-like in their photochemical behavior, exhibiting absorption spectra that are slightly red-shifted with respect to that of typical phthalocyanines and have large extinction coefficients and high fluorescence quantum yields. The free base low symmetry BzIMPAs exhibit unusual fluorescence behavior as a result of a combination of differential annulation along the N-N axes and tautomerism of the species. 


\section{References:}

(1) (a) data for $(t-\mathrm{Bu})_{4} \mathrm{H}_{2} \mathrm{Pc}$ and $(t \text {-Bu })_{4} \mathrm{MgPc}$ : Teuchner, K.; Pfarrherr, A.; Stiel, H.; Freyer, W.; Leupold, D. Photochem. Photobiol. 1993, 57, 465-471. (b) additional data for (t-Bu) ${ }_{4} \mathrm{MgPc}$ : Freyer, W.; Dähne, S.; Minh, L. Q.; Teuchner, K. Z. Chem. 1986, 26, 334-336. (c) data for (t-Bu)4ZnPc: TranThi, T.-H.; Desforge, C.; Thiec, C.; Gaspard, S. J. Phys. Chem. 1989, 93, 1226-1233.

(2) Kobayashi, N.; Mack, J.; Ishii, K.; Stillman, M. J. Inorg. Chem. 2002, 41, 5350-5363. 


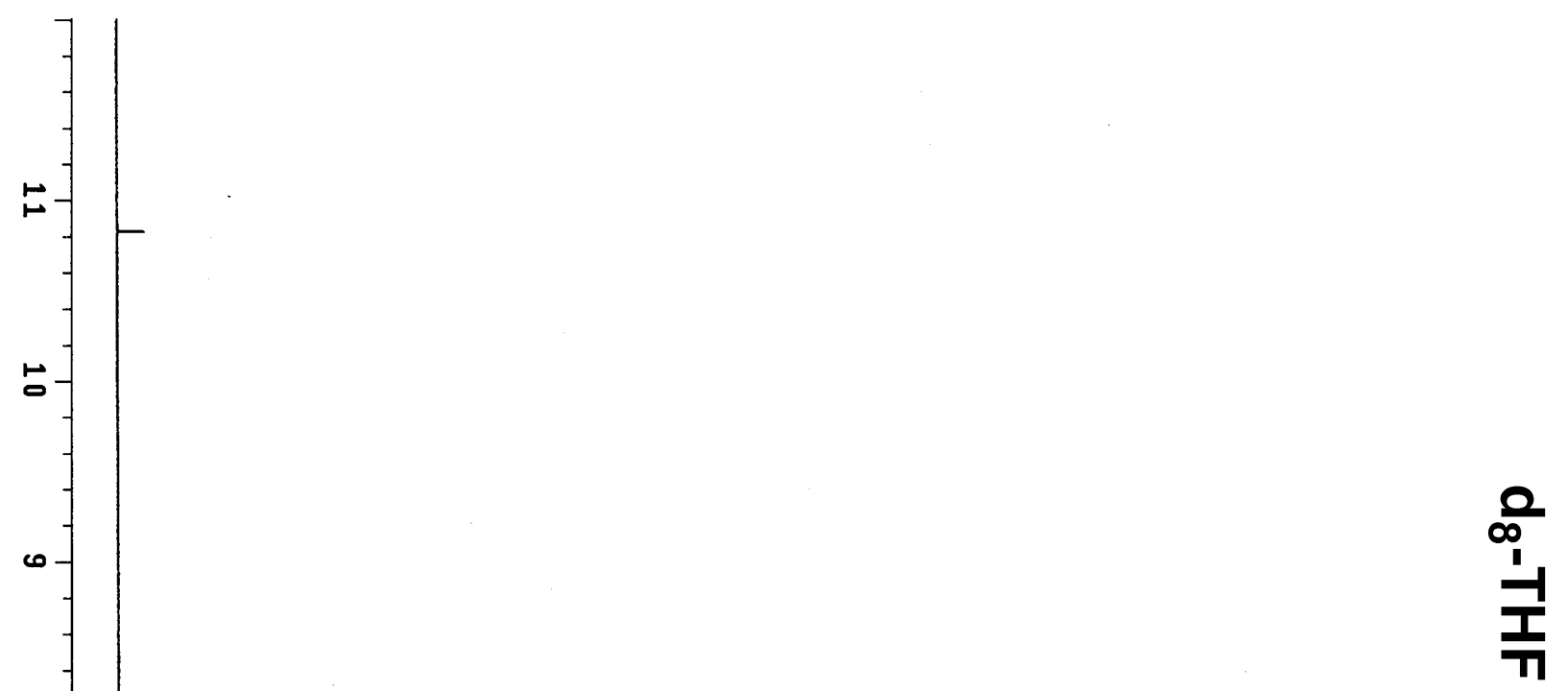

$\infty-$

o

$-1$

ज-

$\rightarrow-$

$\omega$

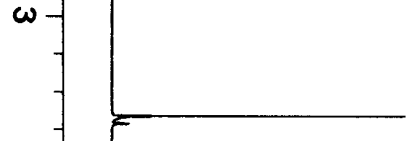

$N-$

每-

S 10 


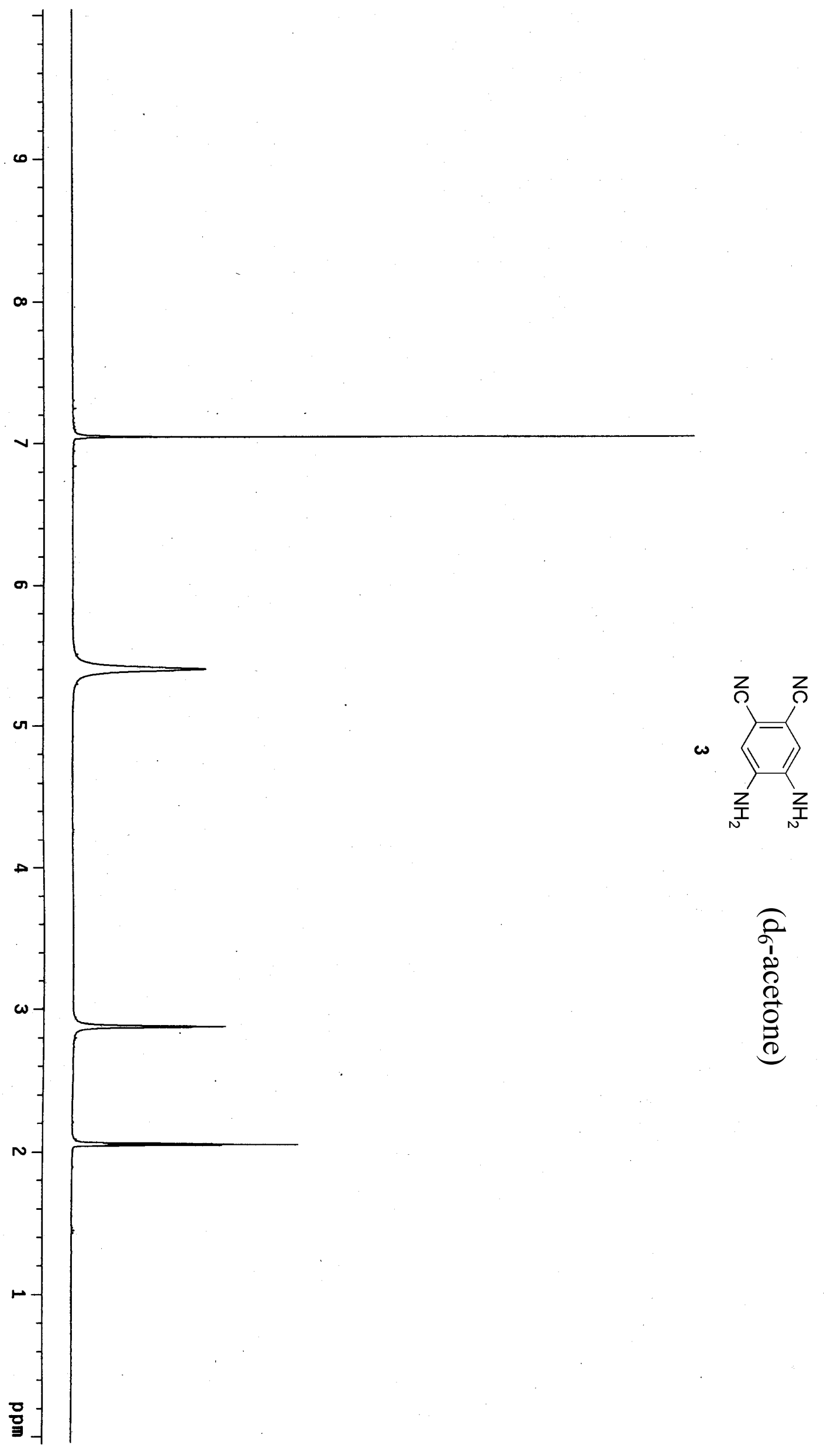




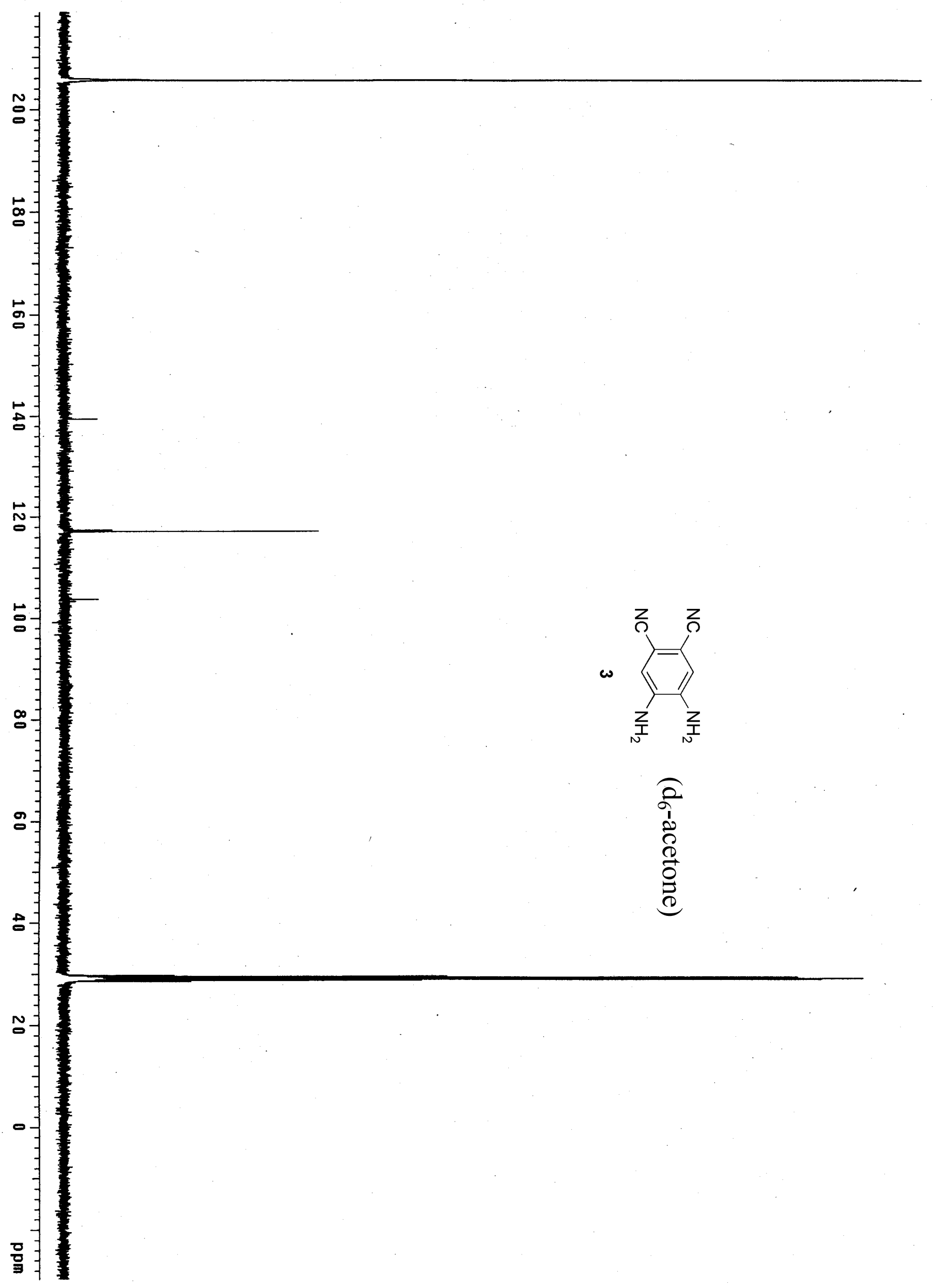




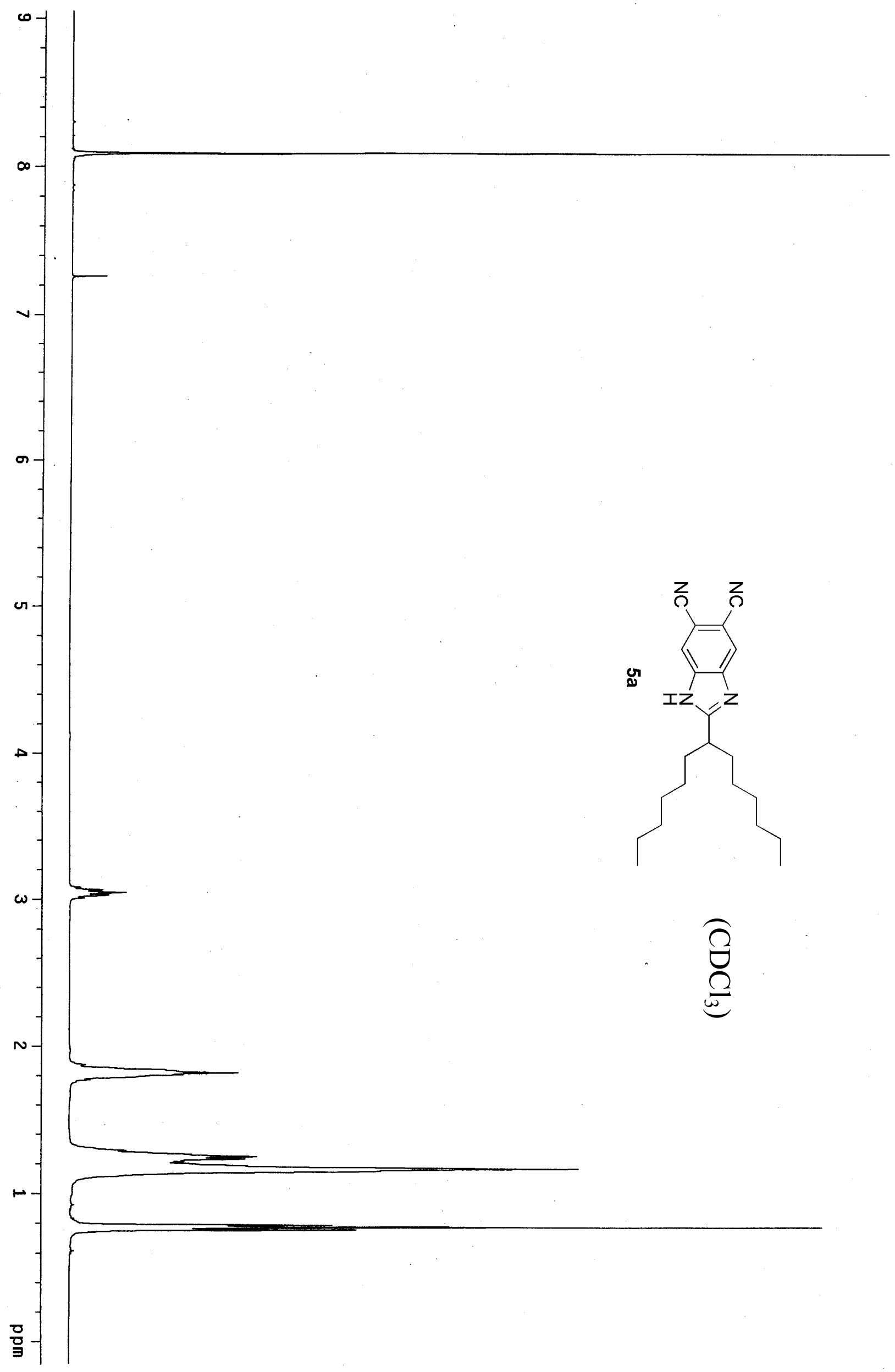

S 13 


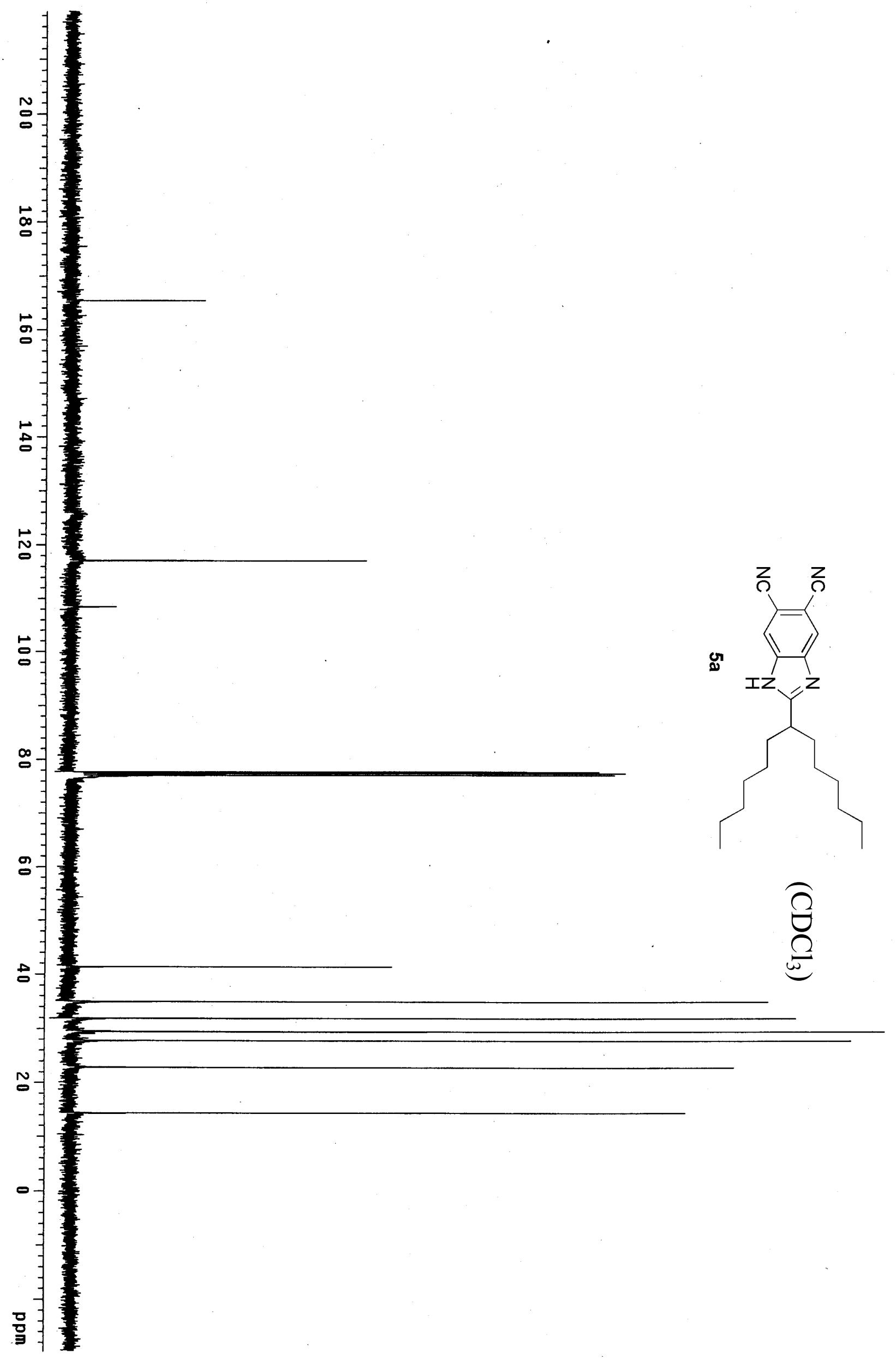

S 14 


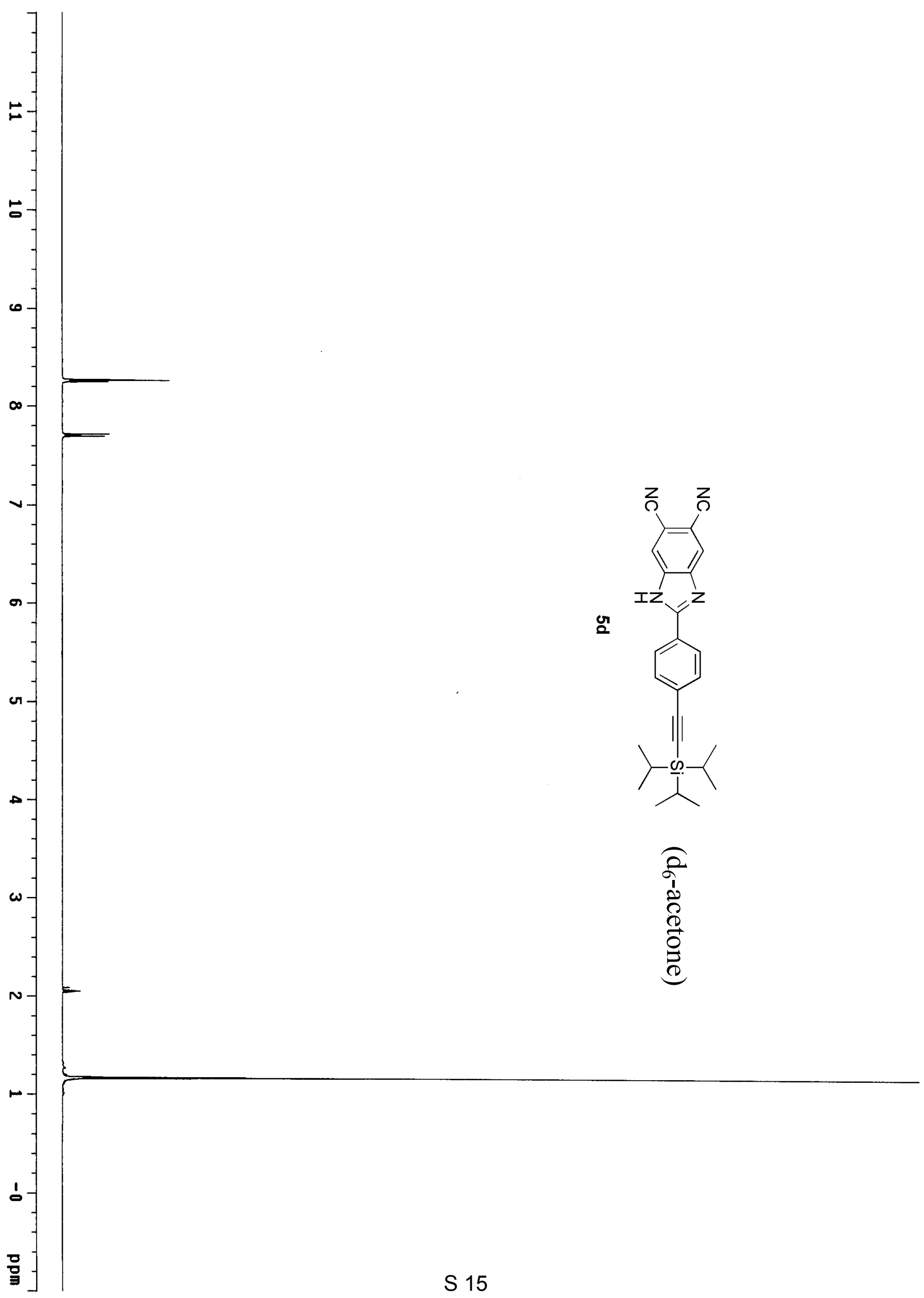




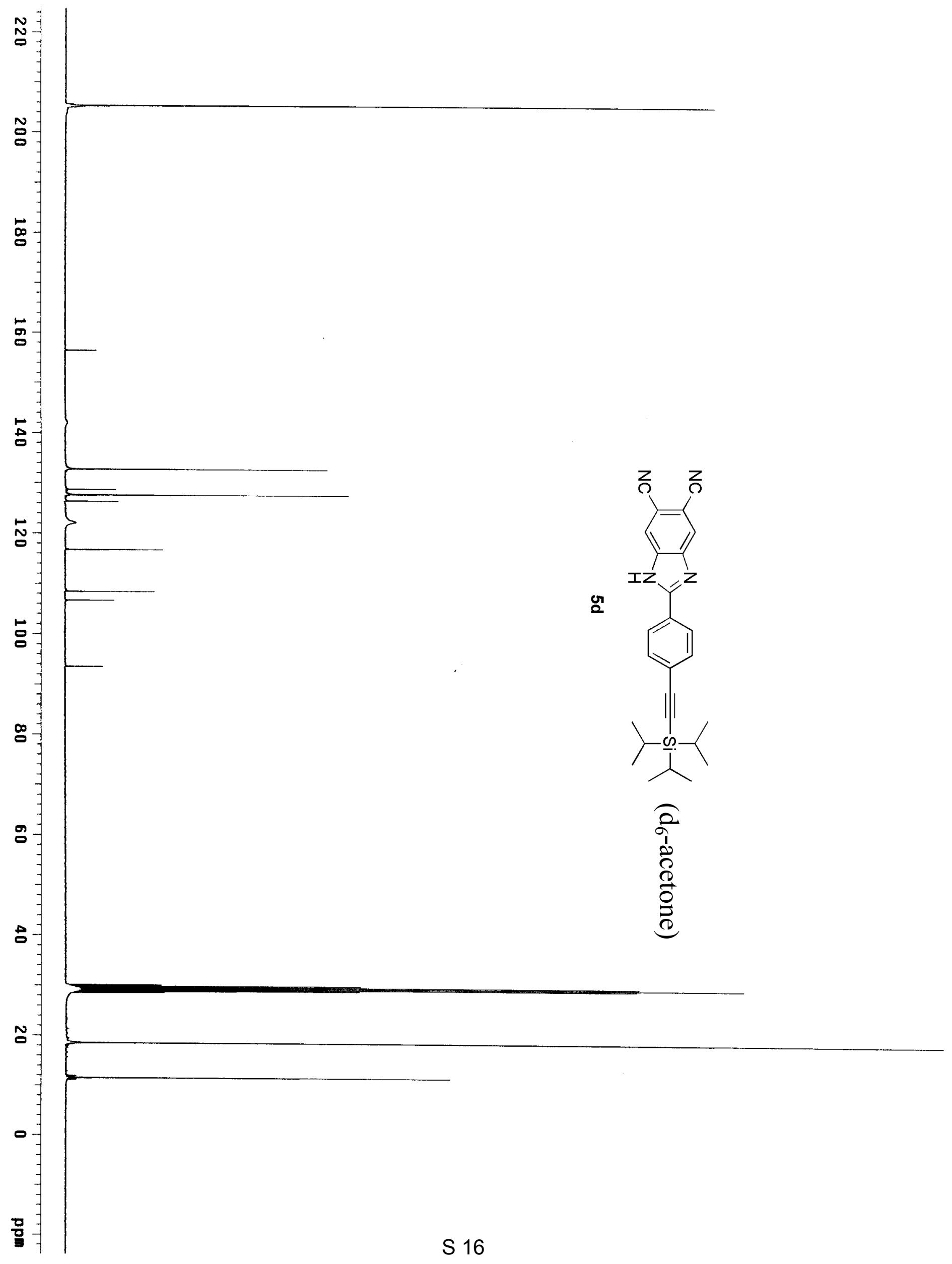




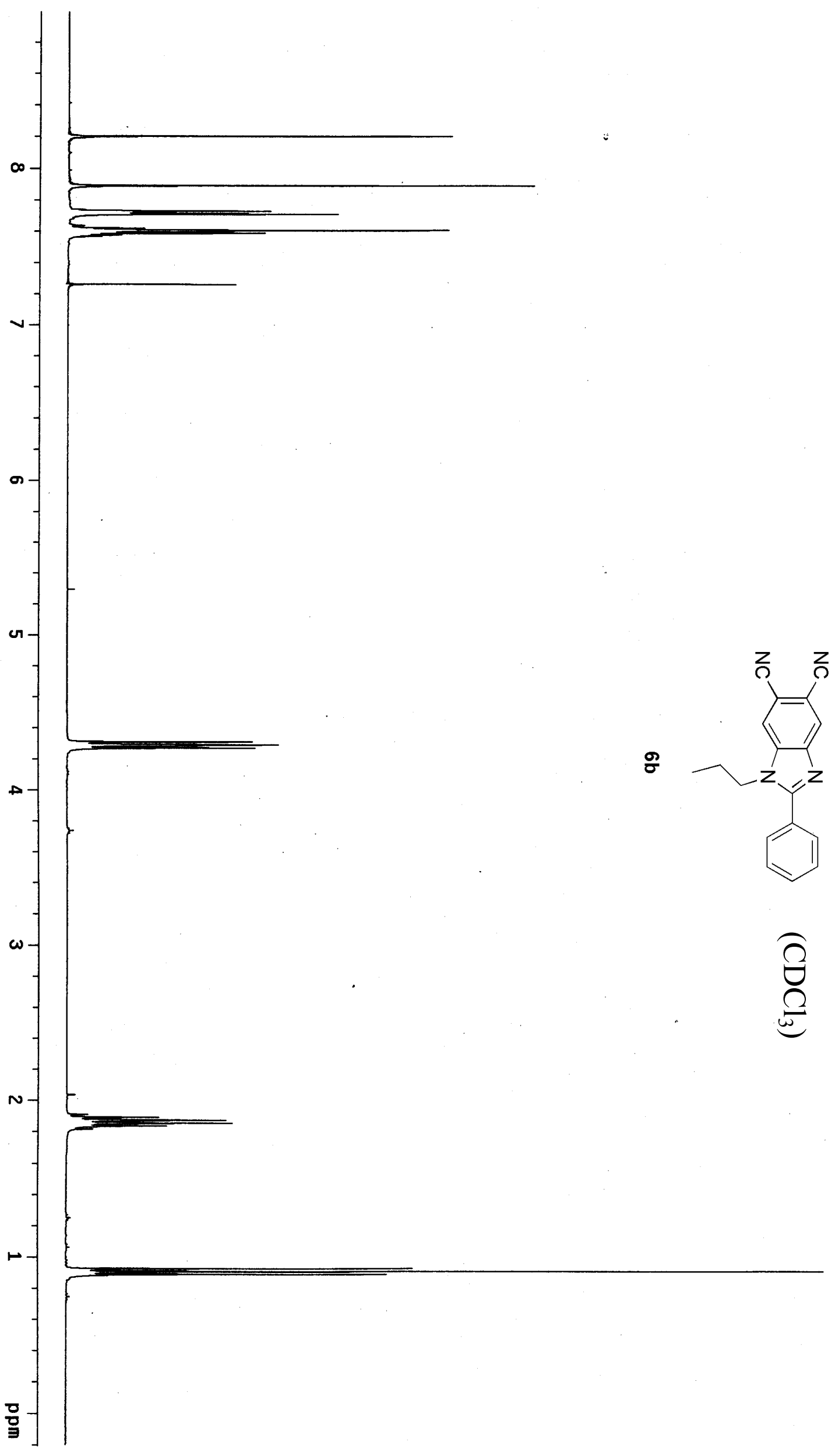




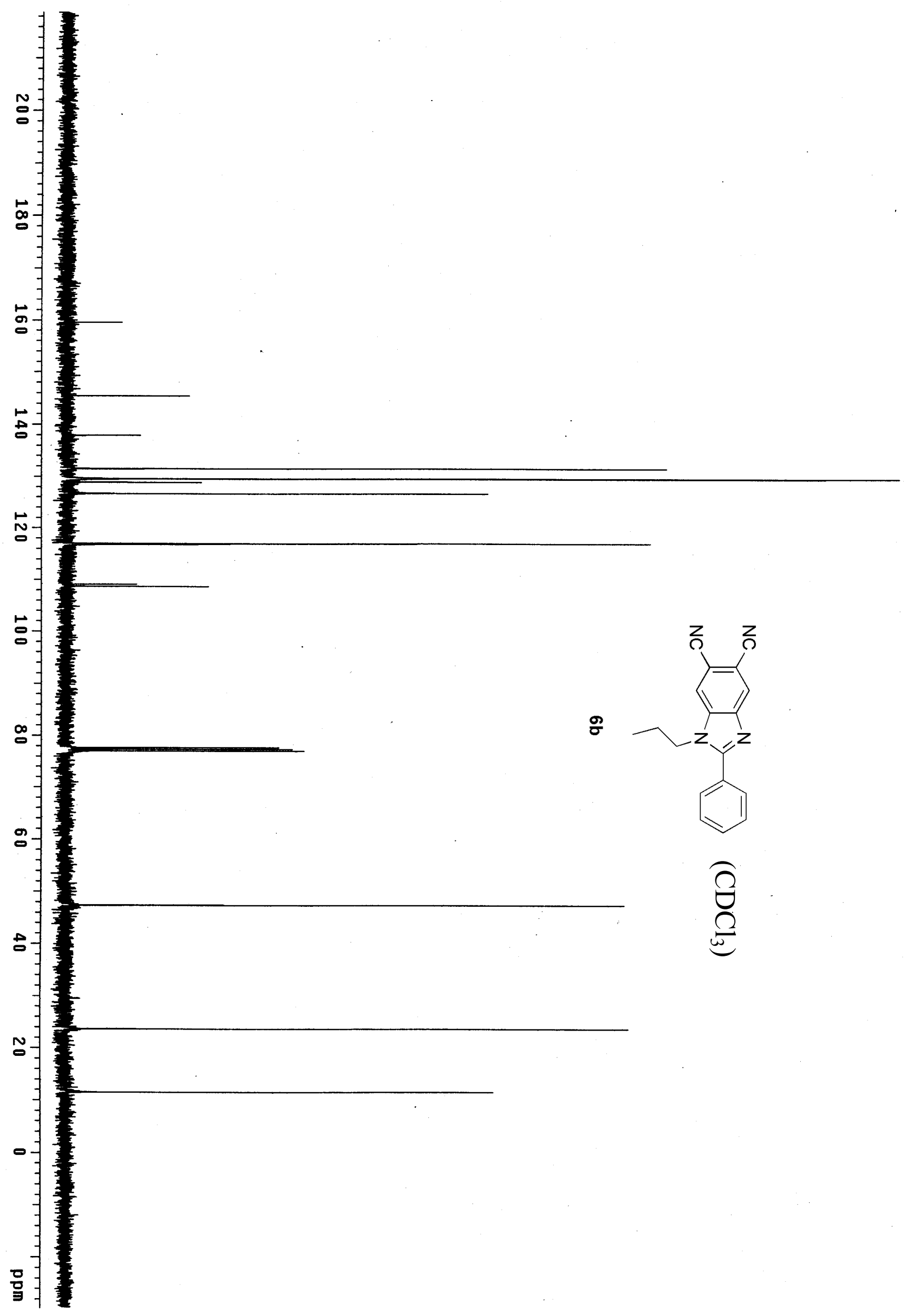

S 18 


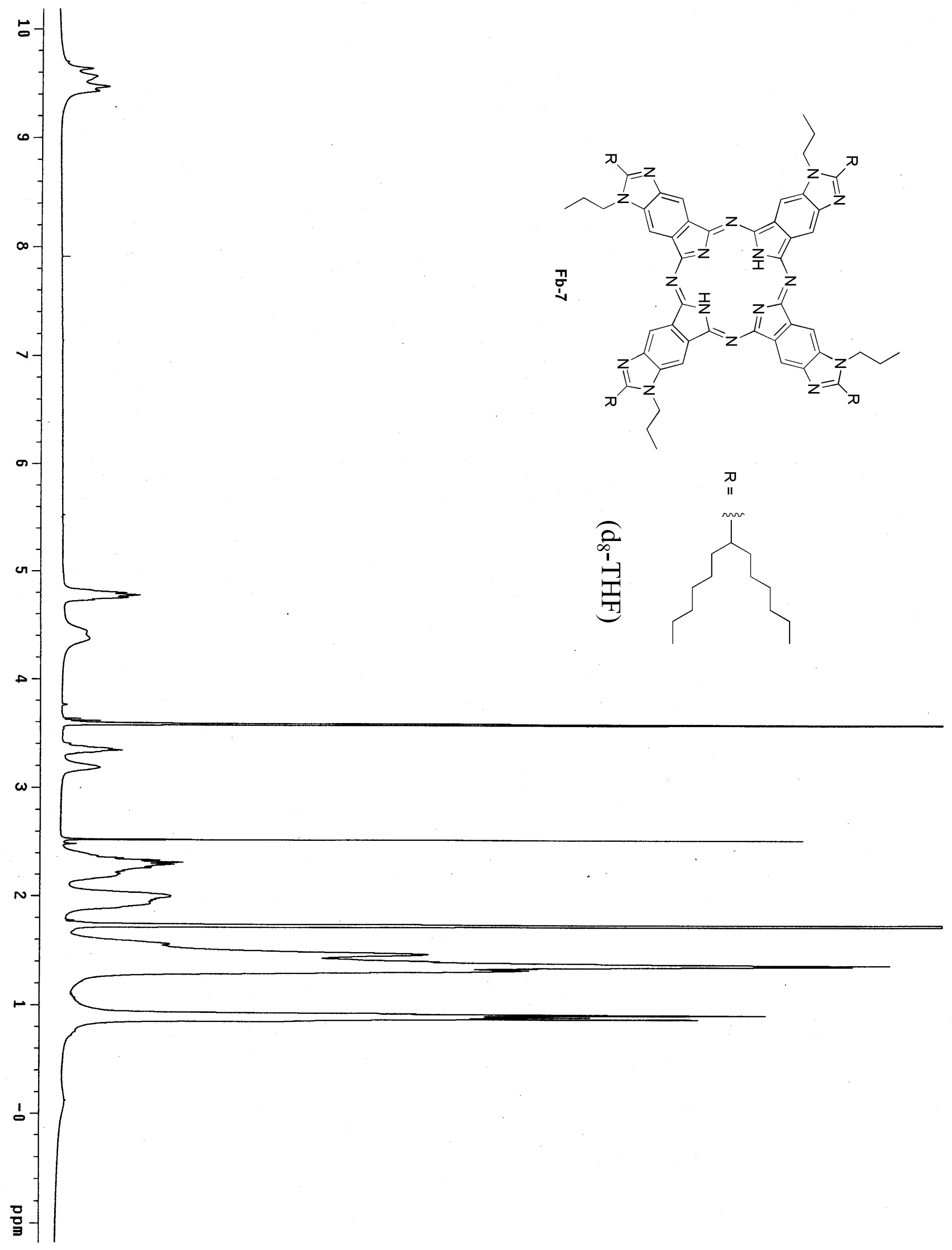




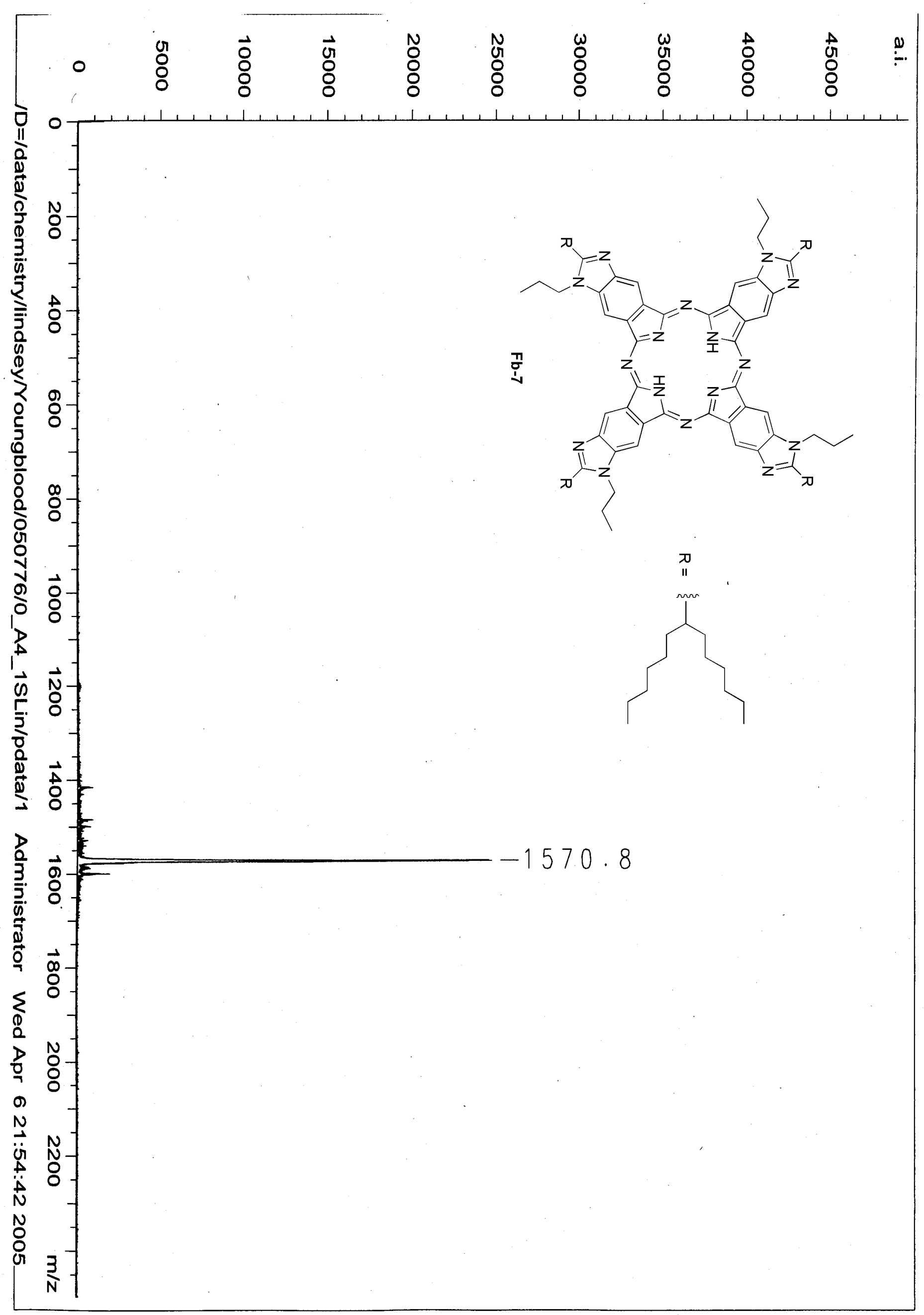




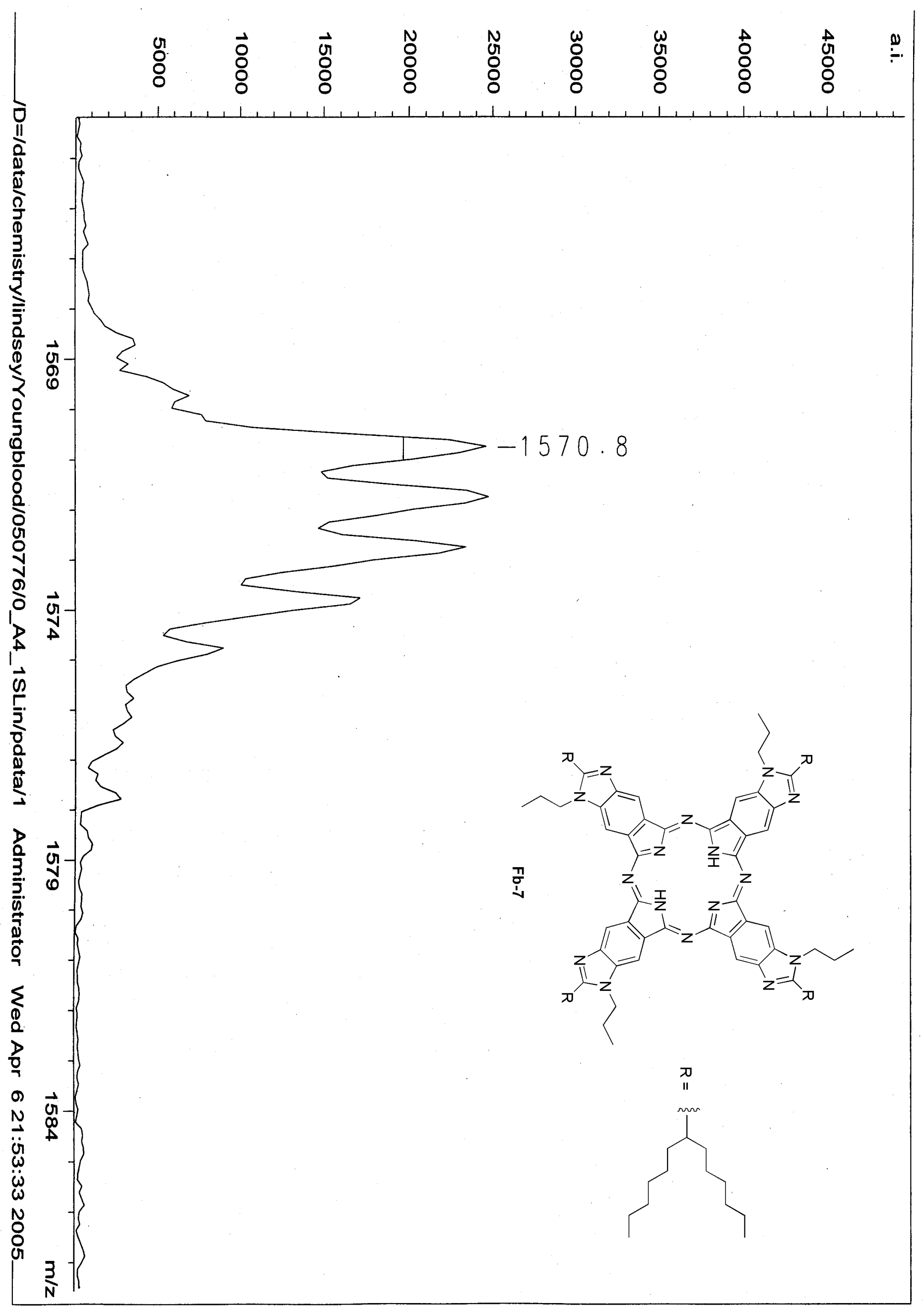


Method file: Information : Data File : <untitled>

Default Method

C: \JUSTIN \5-9-05\FBSWT4 PA.SD Created : 5/9/05

$17: 47: 37$

Overlaid Spectra:

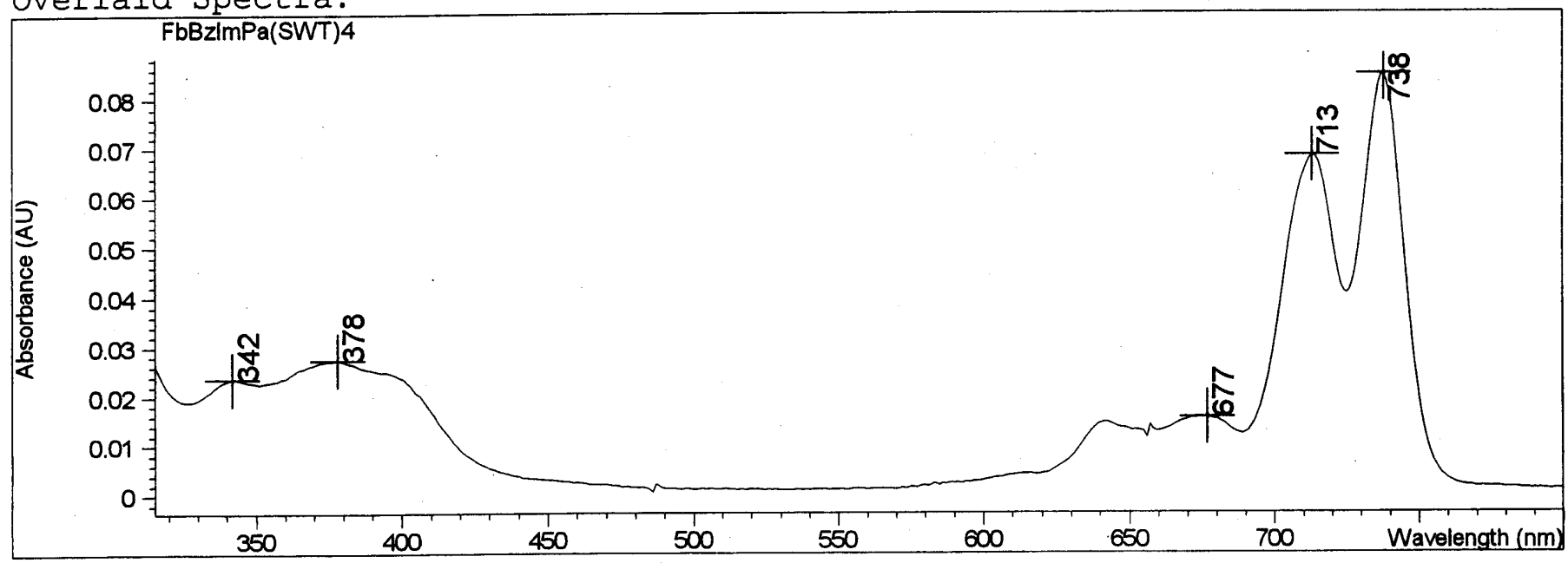

\begin{tabular}{lrrr}
$\#$ & Name & Peaks (nm) & Abs (AU) \\
\hline 1 & FbBzImPa (SWT) 4 & 738.0 & $8.4637 \mathrm{E}-2$ \\
1 & & 713.0 & $6.8223 \mathrm{E}-2$ \\
1 & 378.0 & $2.7367 \mathrm{E}-2$ \\
1 & 342.0 & $2.3540 \mathrm{E}-2$ \\
1 & 677.0 & $1.5609 \mathrm{E}-2$
\end{tabular}

Report generated by :

Signature:

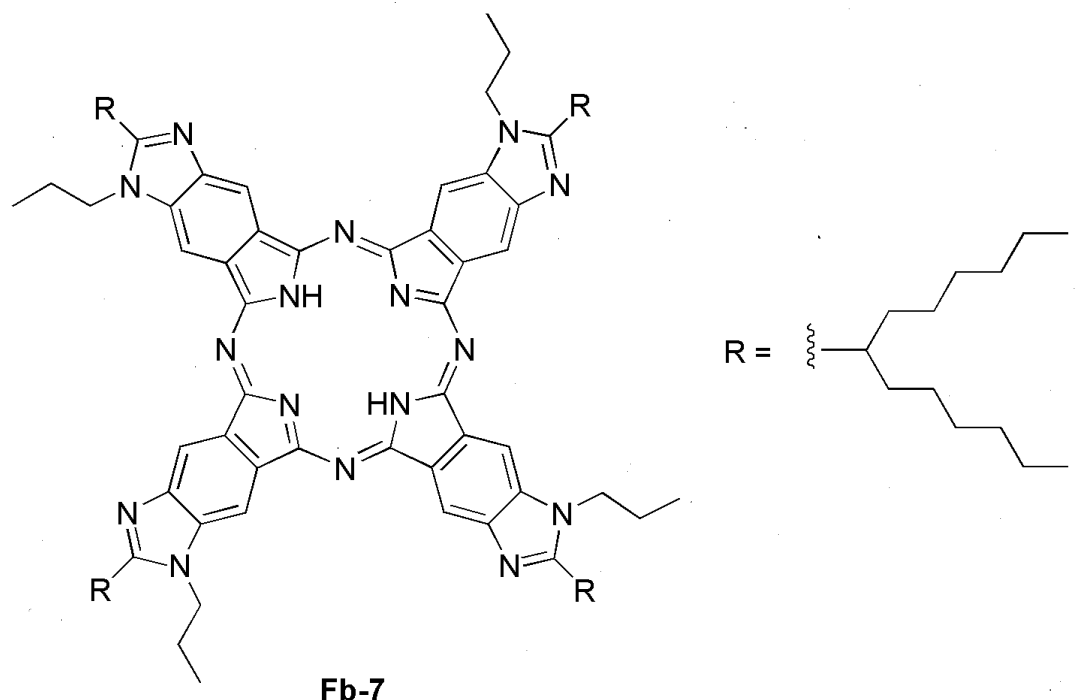




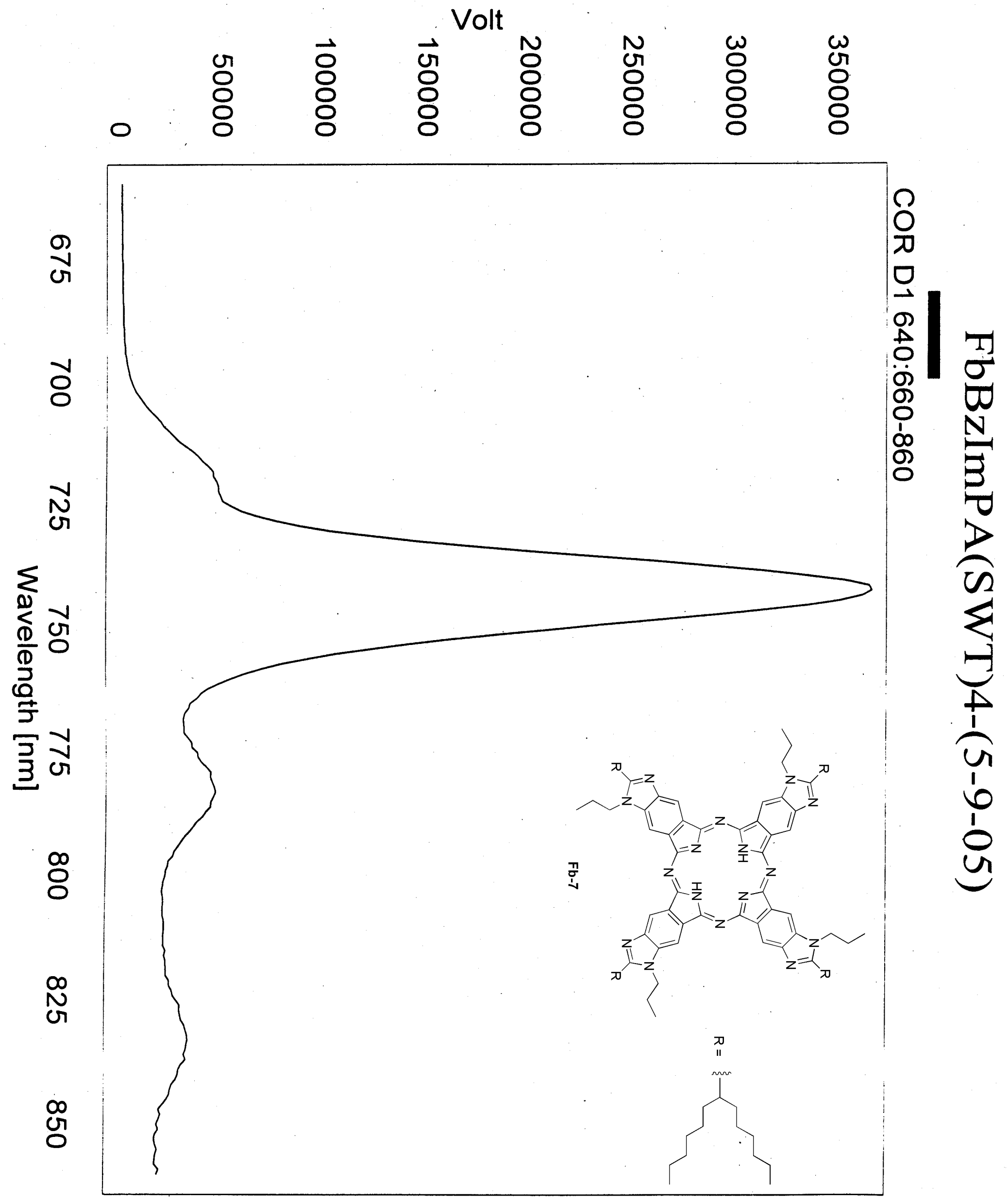




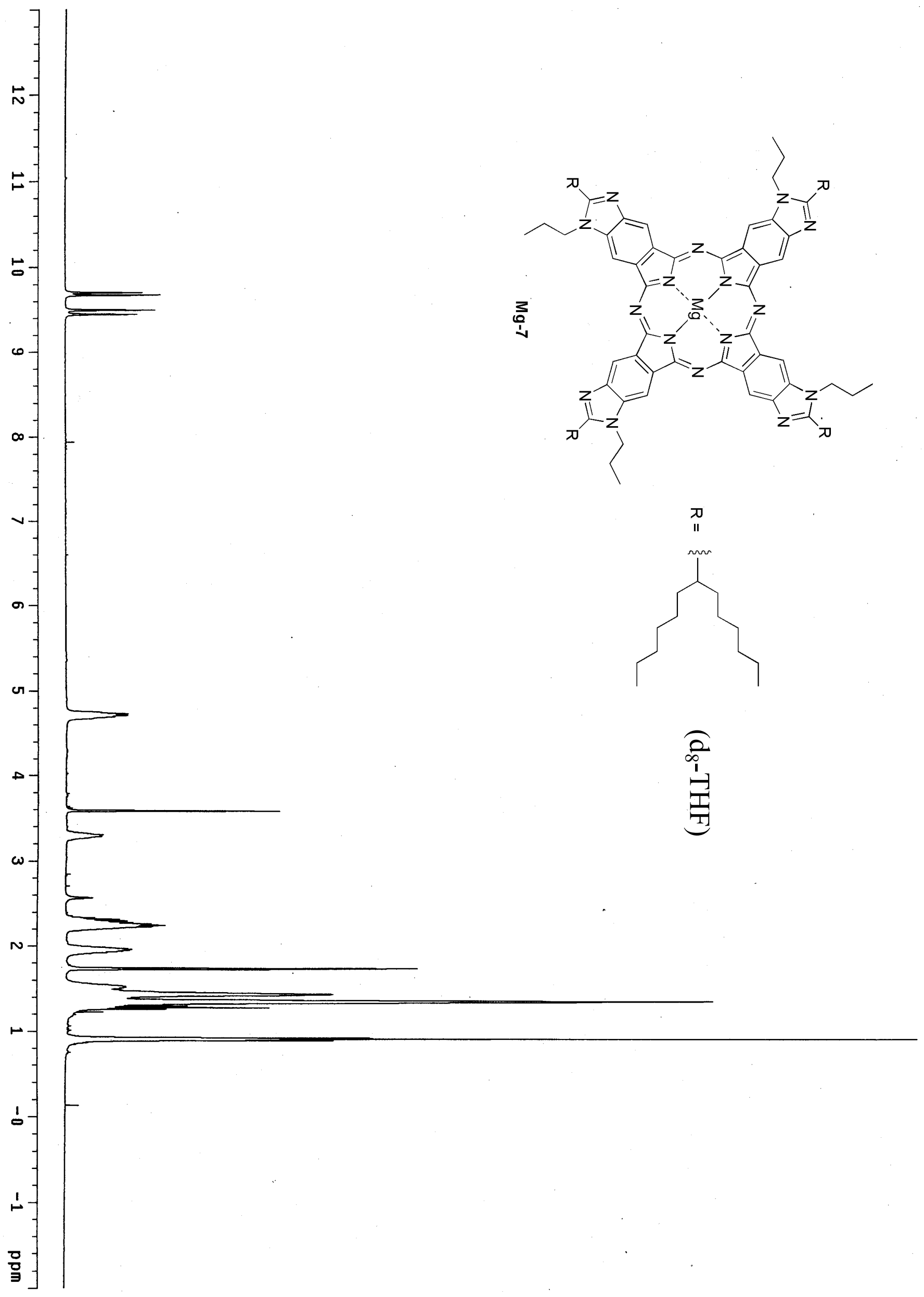




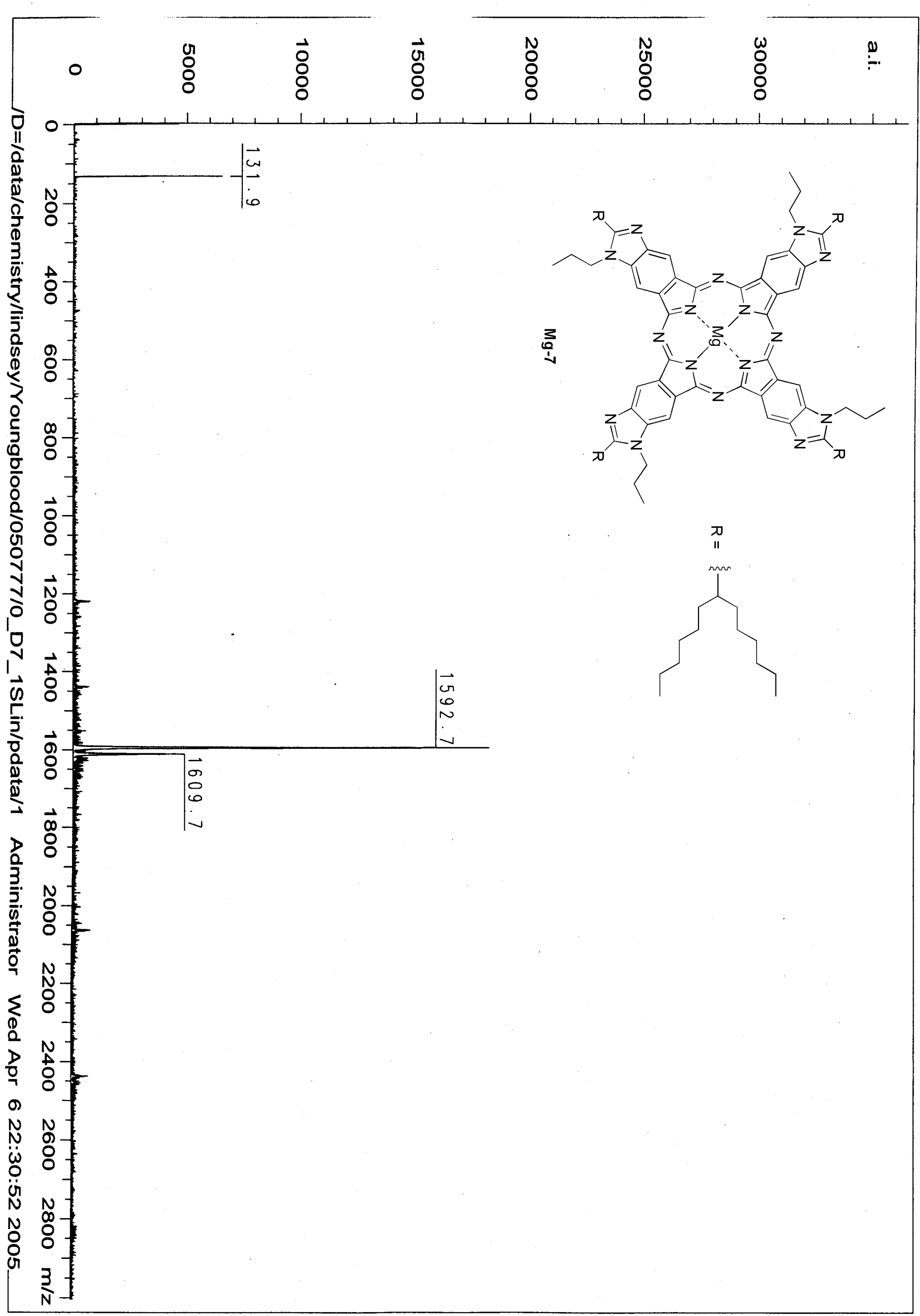


Method file : Information : Data File : <untitled>

Default Method

C: \JUSTIN \5-9-05\MGSWTPA.SD

$17: 14: 15$

Overlaid Spectra:

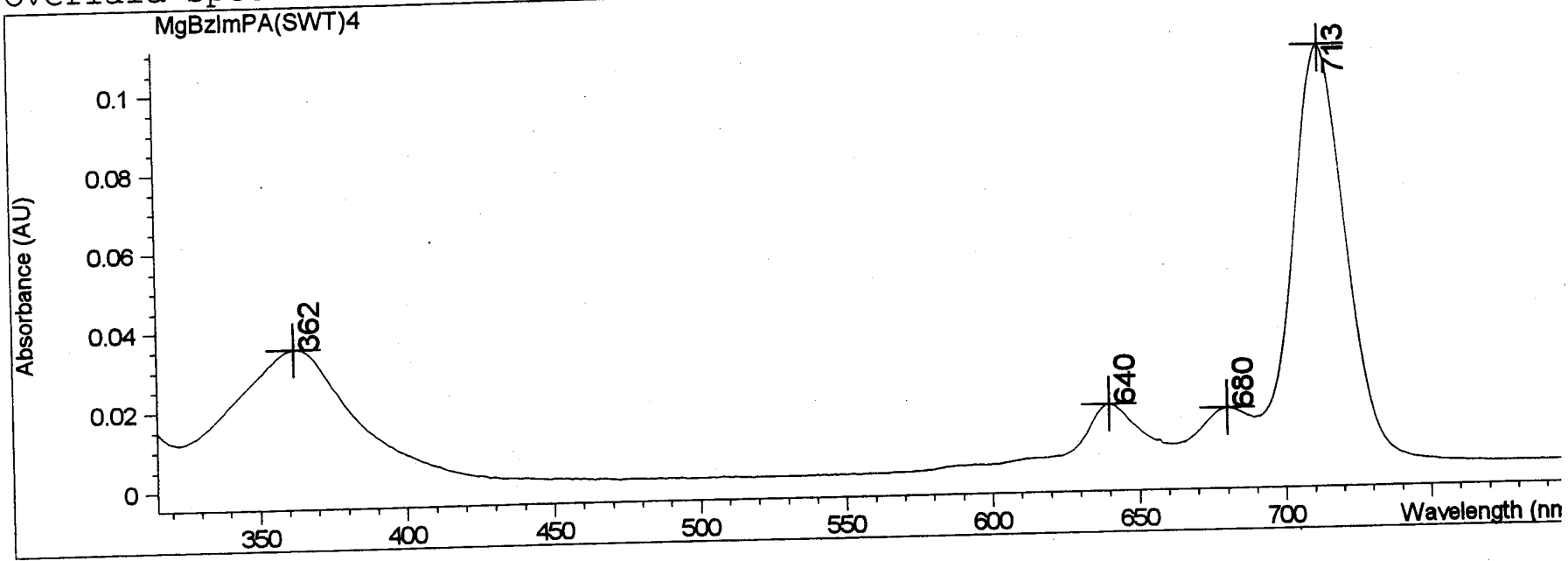

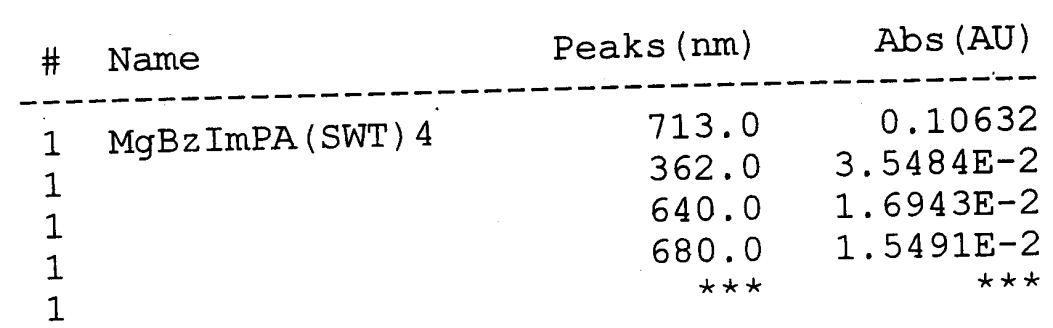

Report generated by :

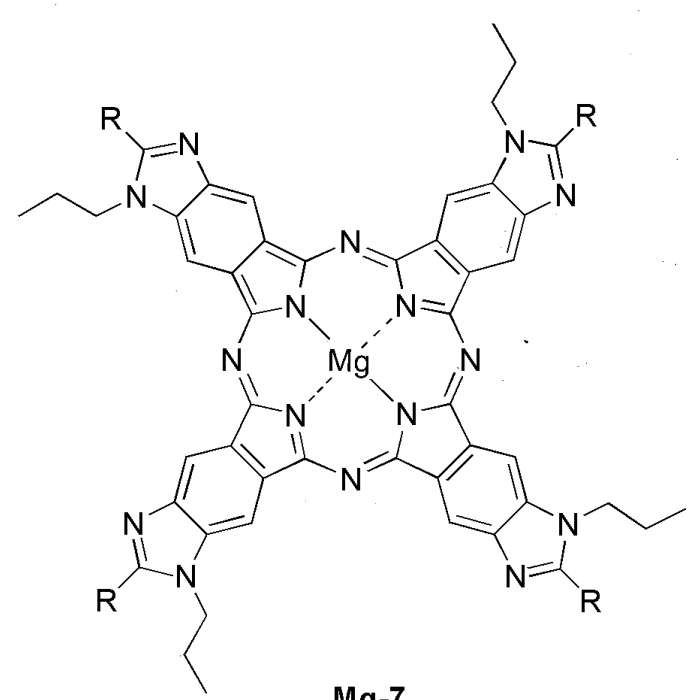

Mg-7
Created : 5/9/05 


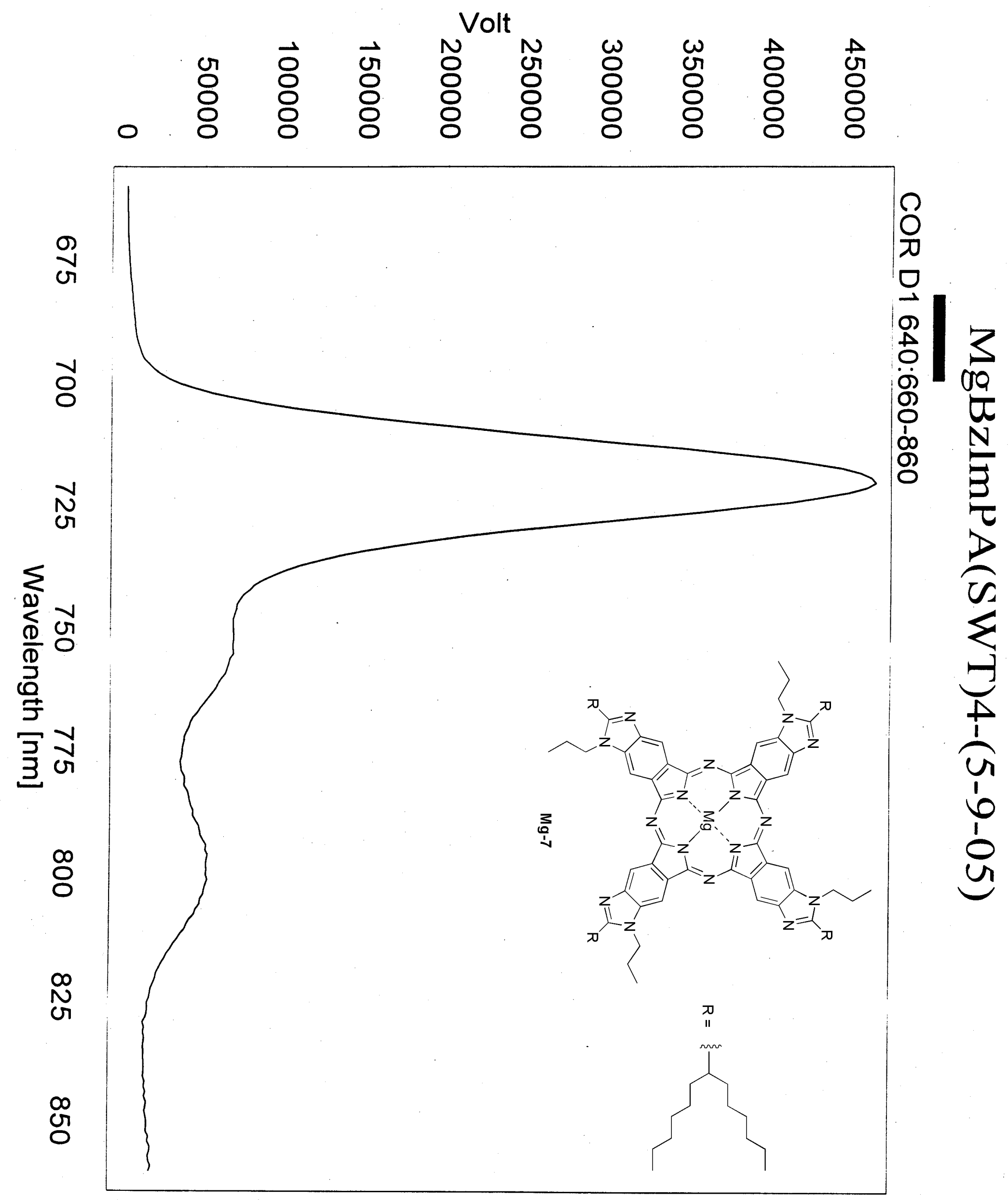




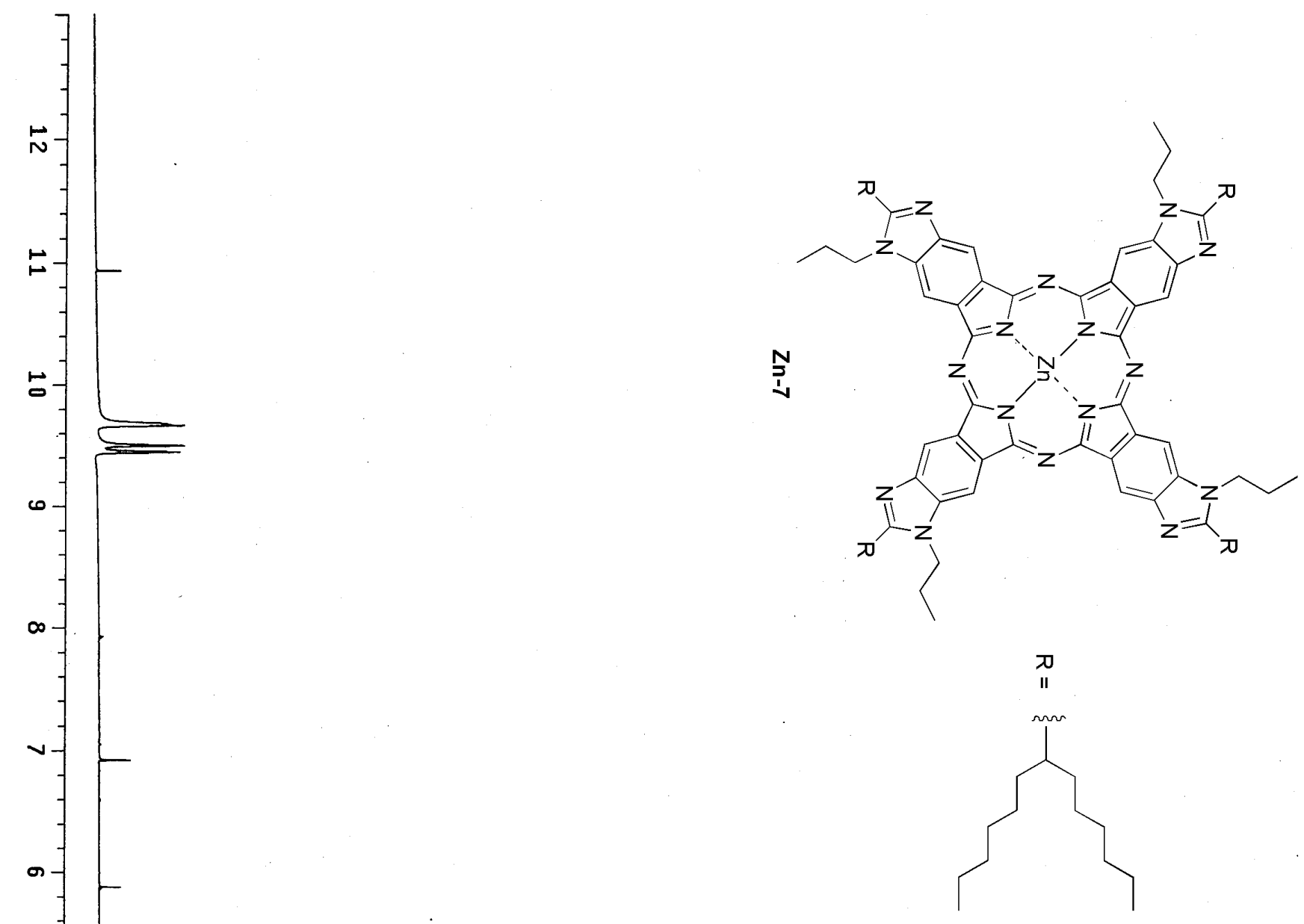

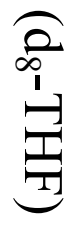




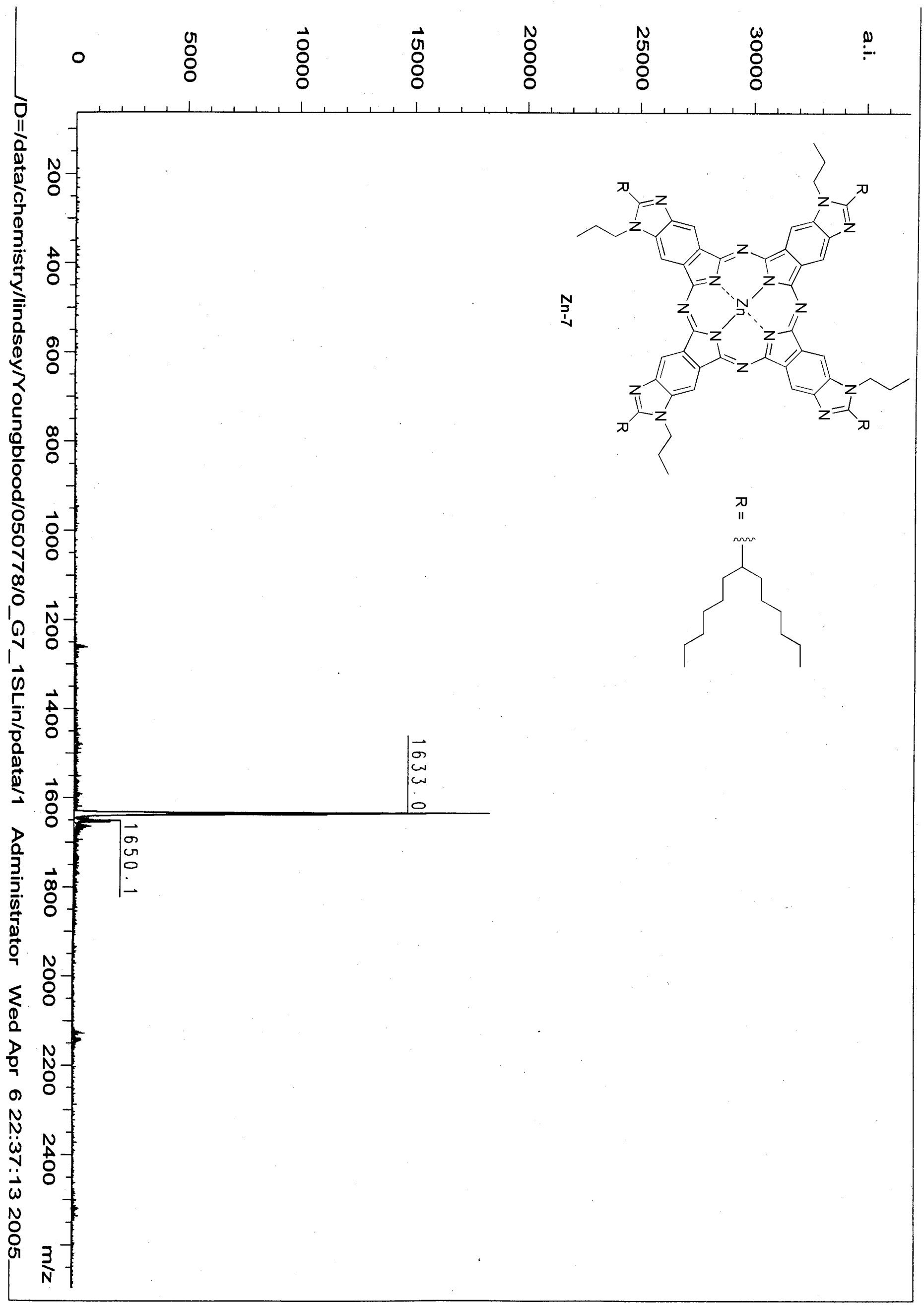


Method file: Information : Data File : <untitled>

Default Method

C: \JUSTIN \5-9-05\ZNSWT 4 PA.SD

$15: 52: 47$

Overlaid Spectra:

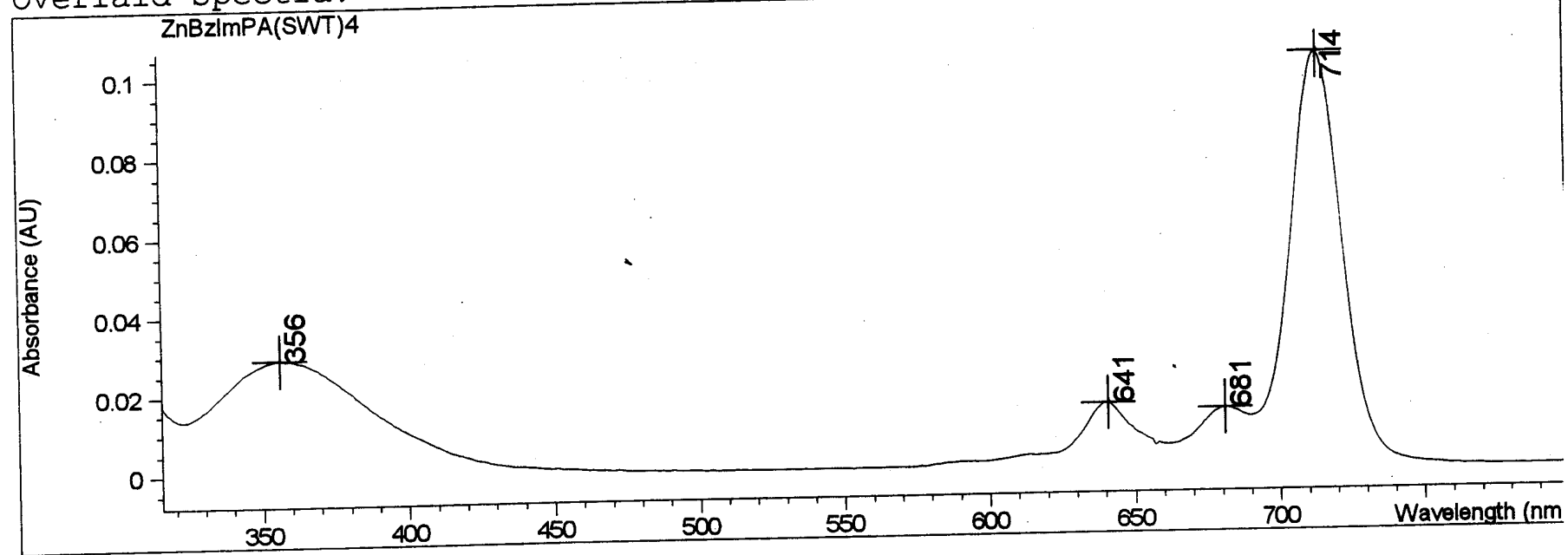

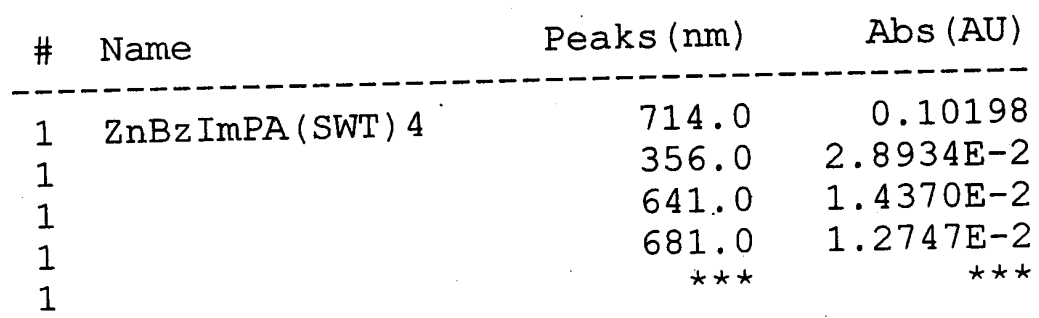

Report generated by :

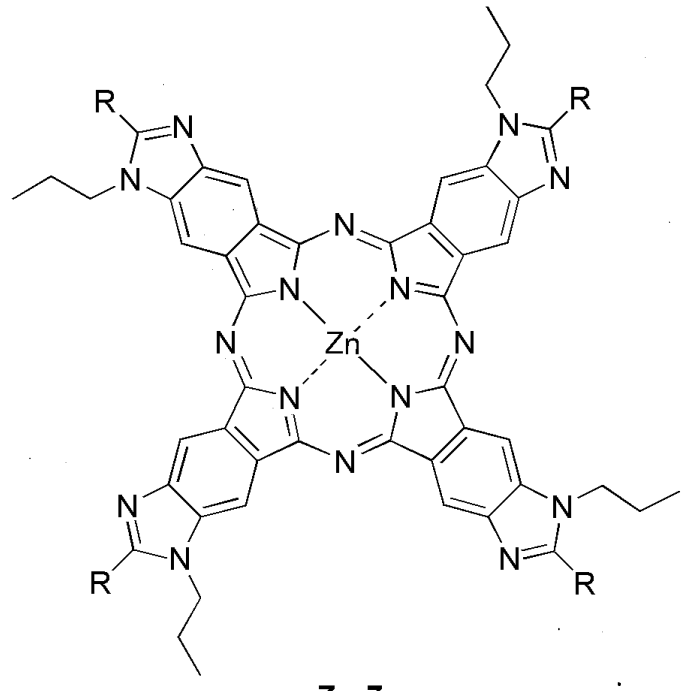

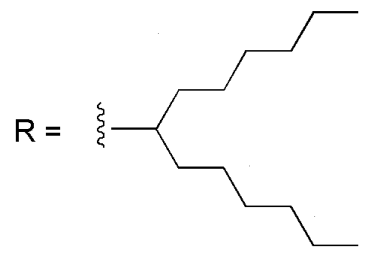

$\mathrm{Zn}-7$ 


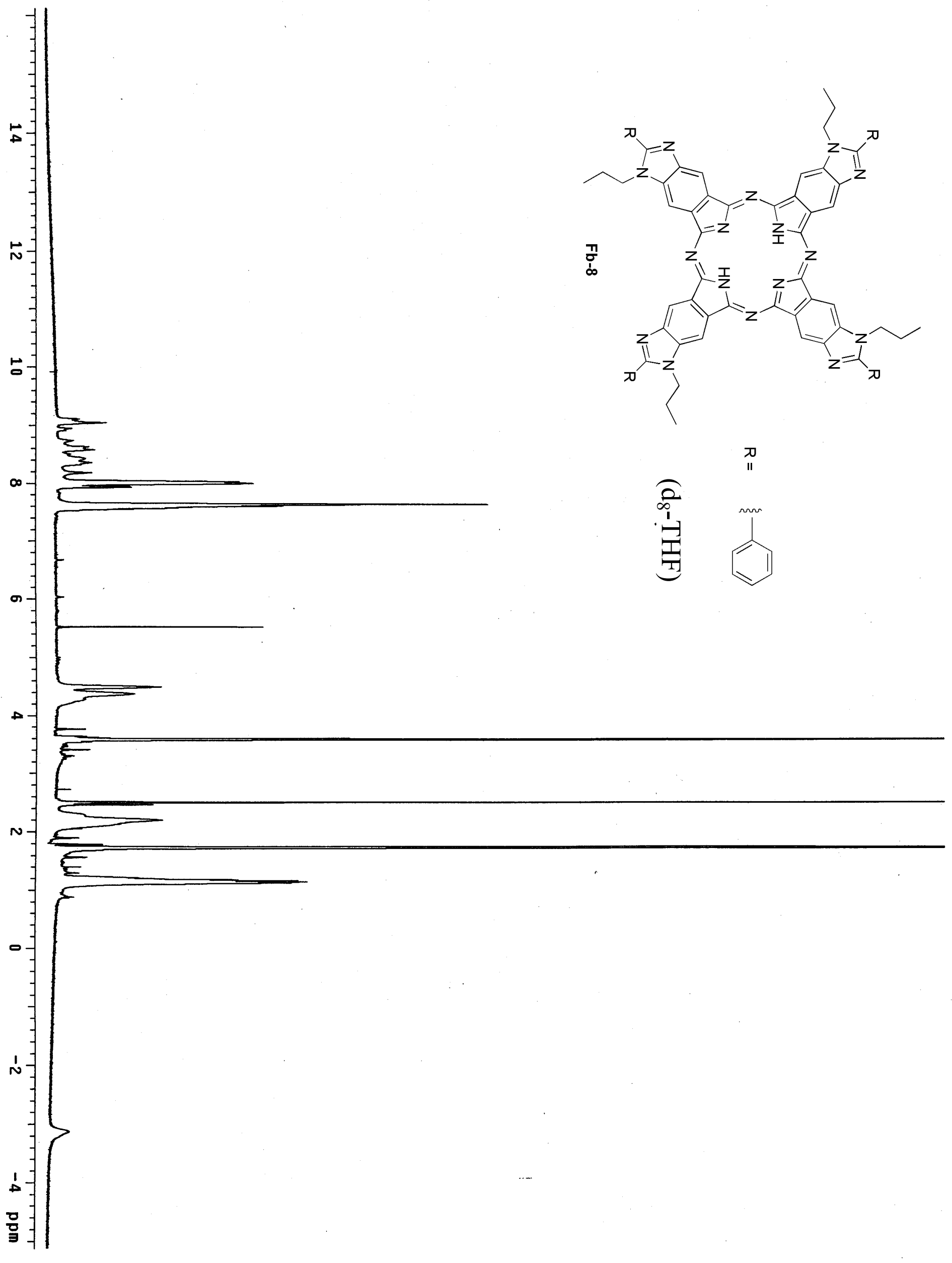




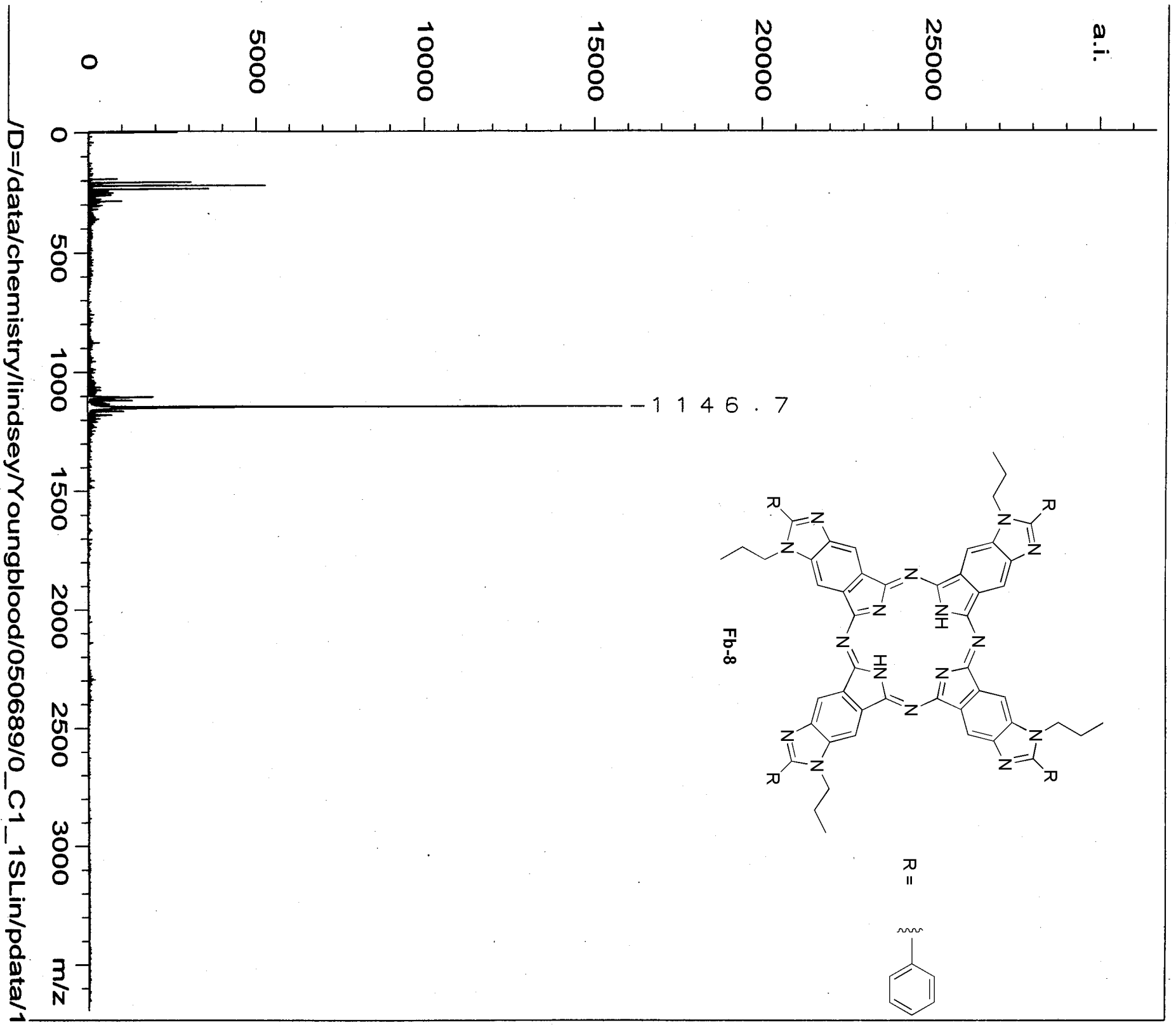

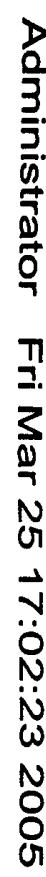

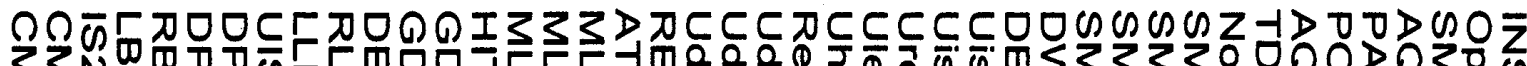

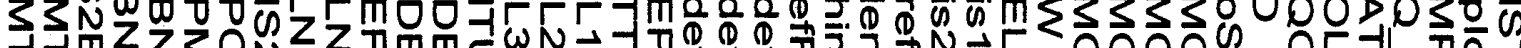

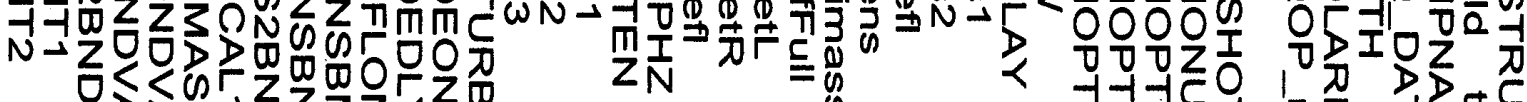

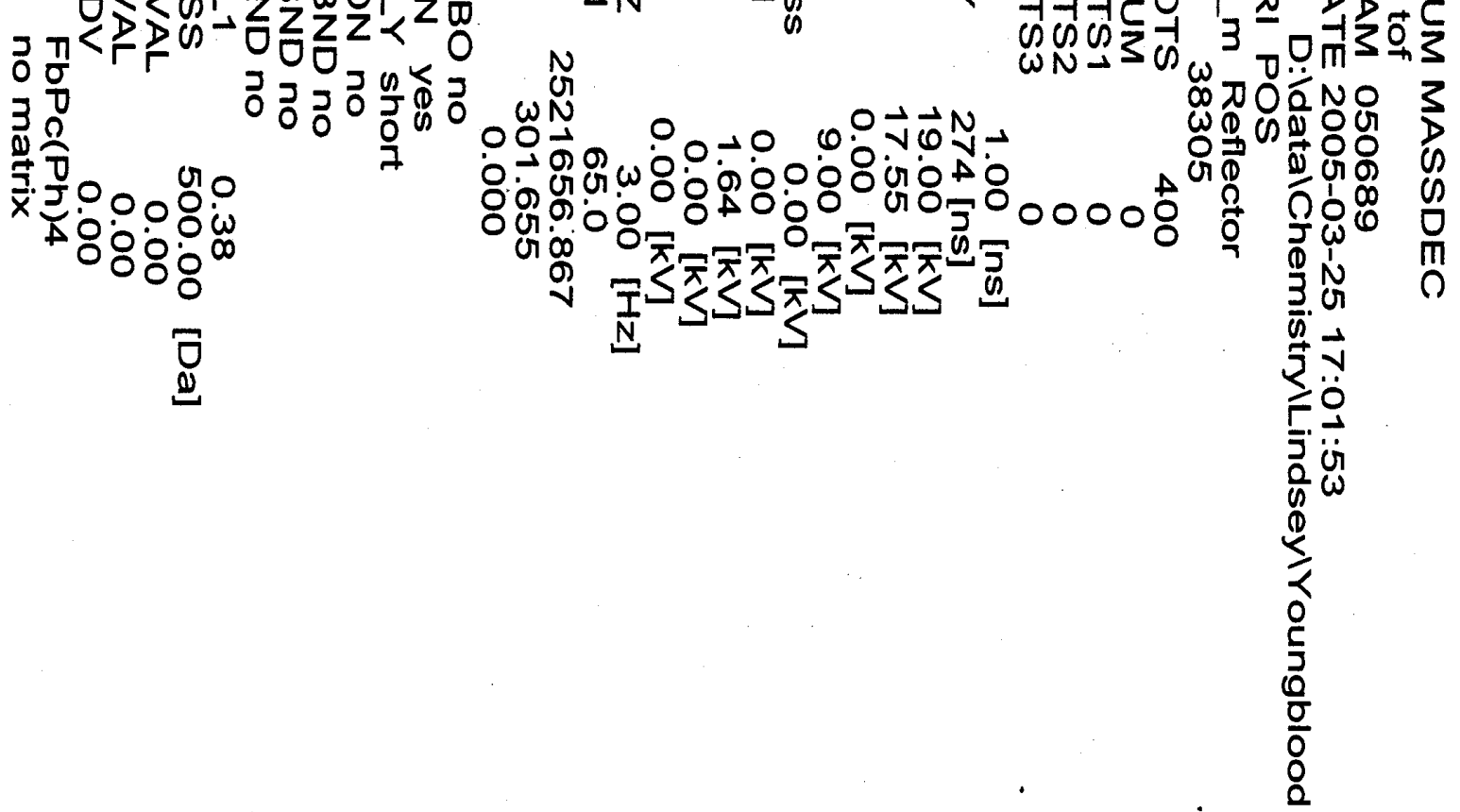



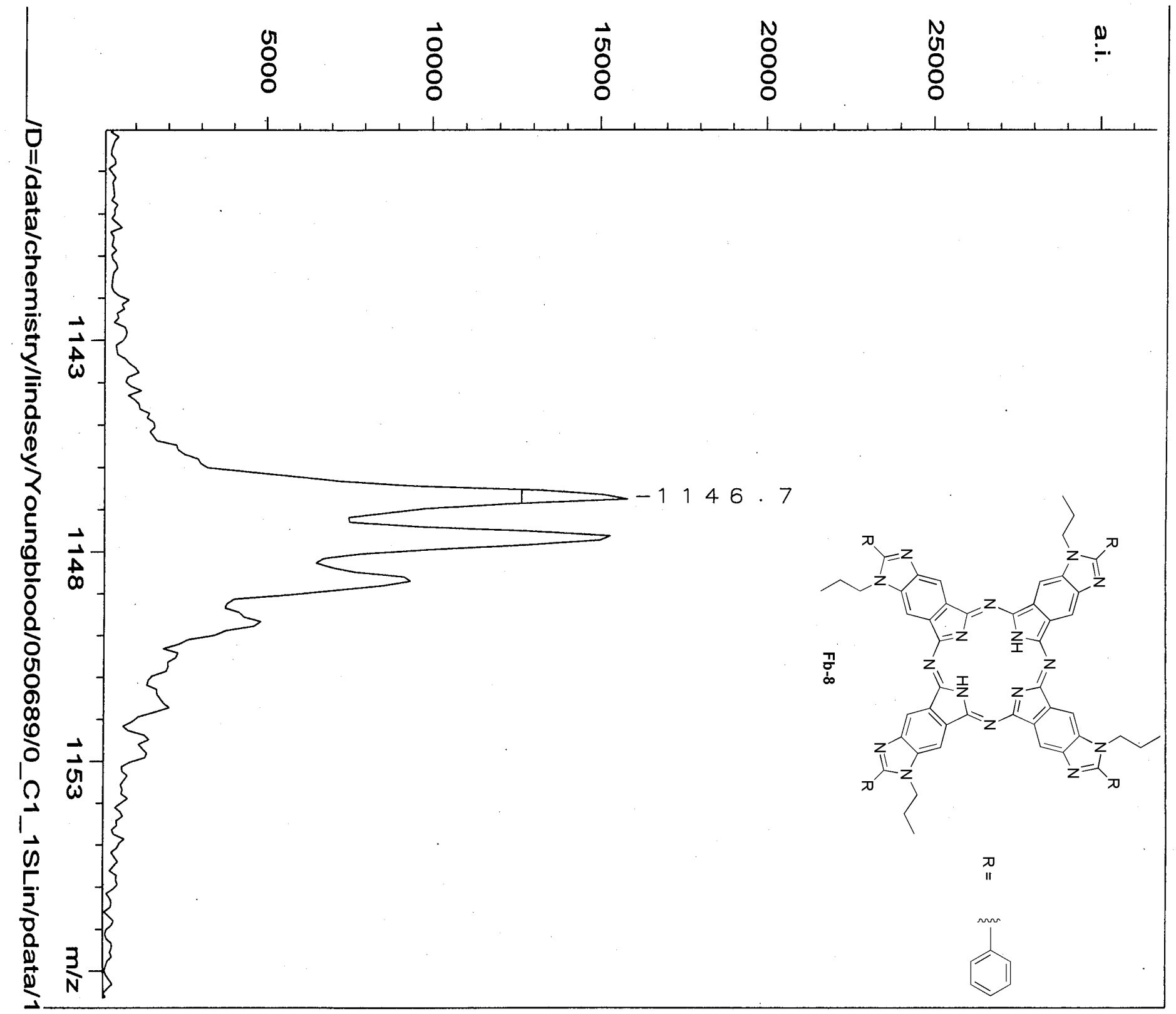

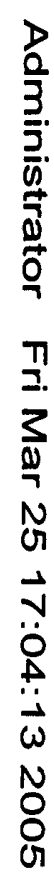

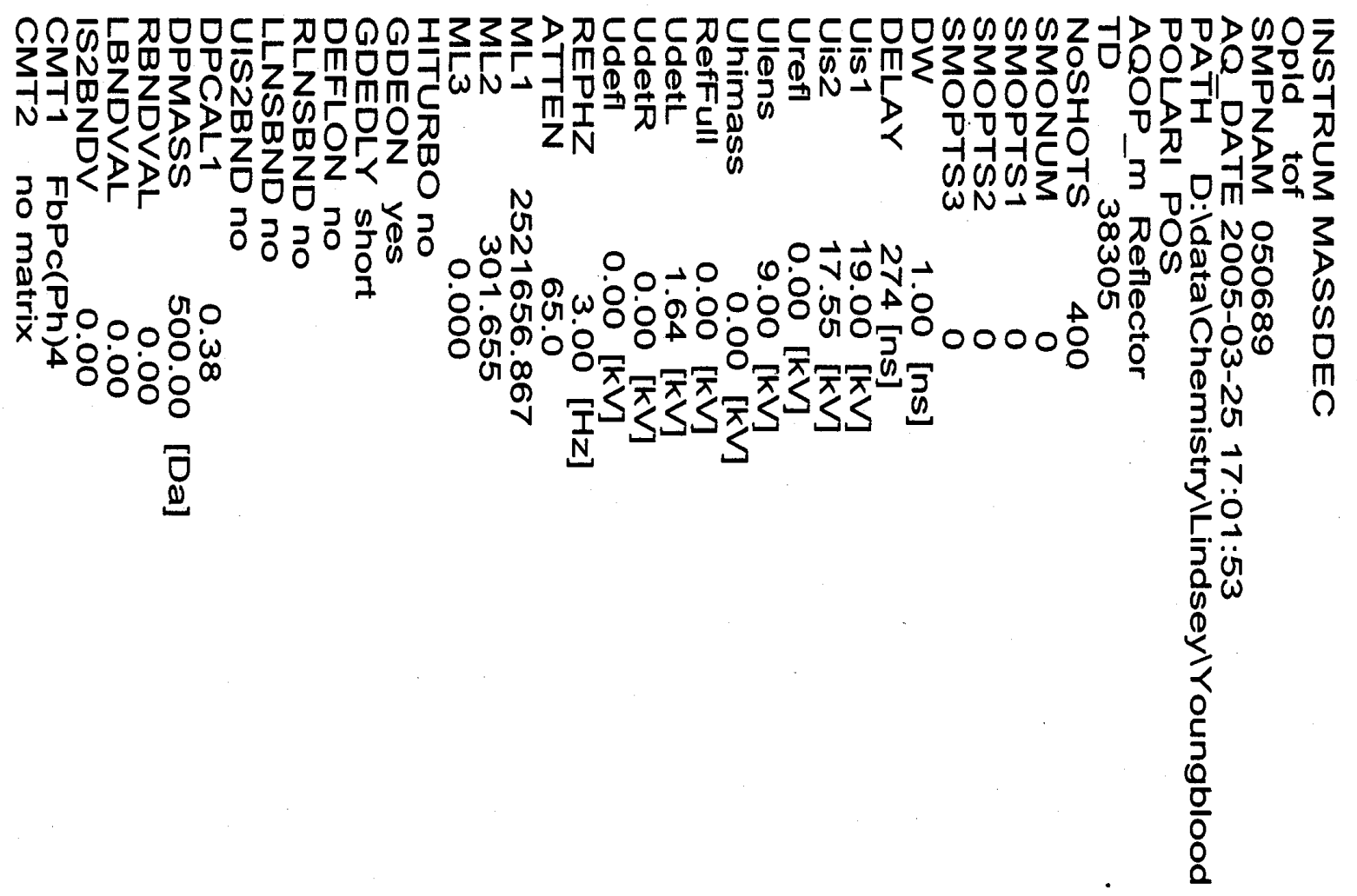


Method file: Information :

Data File : <untitled>

Default Method

C: \UUSTIN \5-9-05\FBPH4PA.SD Created : 5/9/05

$17: 56: 28$

Overlaid Spectra:

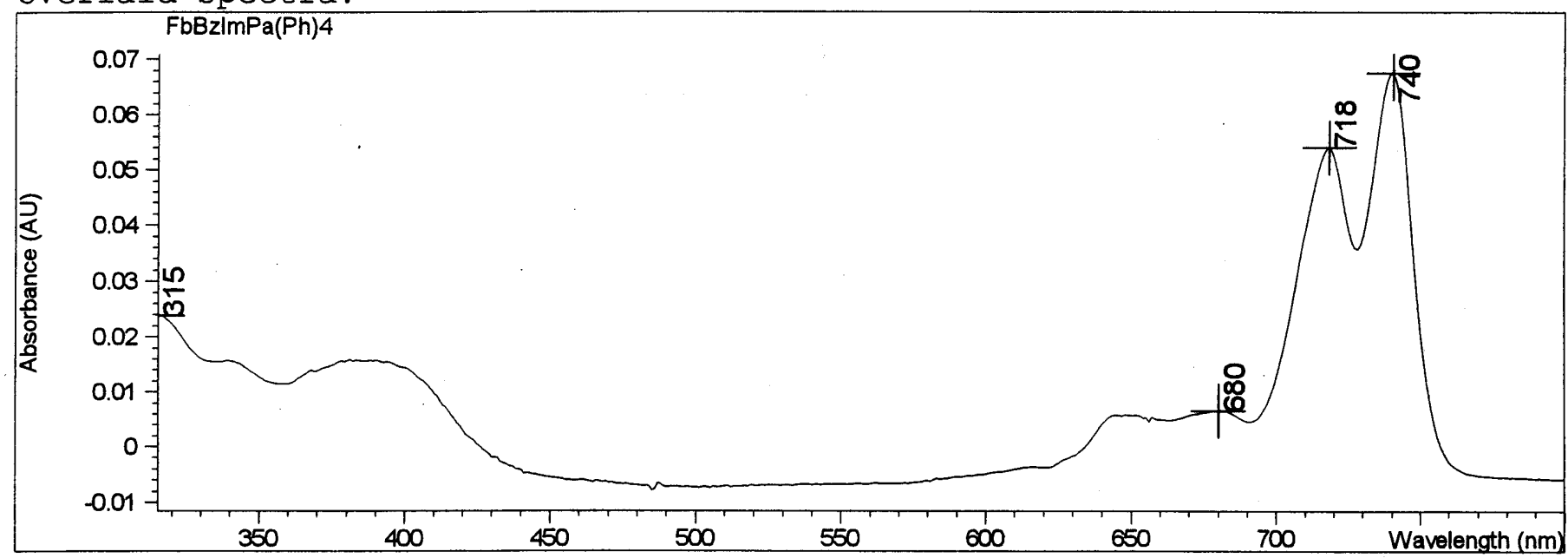

\begin{tabular}{rrrr}
$\#$ & Name & Peaks $(\mathrm{nm})$ & Abs $(\mathrm{AU})$ \\
\hdashline 1 & FbBzImPa (Ph) 4 & 740.0 & $6.7390 \mathrm{E}-2$ \\
1 & & 718.0 & $5.3867 \mathrm{E}-2$ \\
1 & 315.0 & $2.3790 \mathrm{E}-2$ \\
1 & 680.0 & $6.5460 \mathrm{E}-3$ \\
1 & $\star \star *$ & $\star \star *$
\end{tabular}

Report generated by :

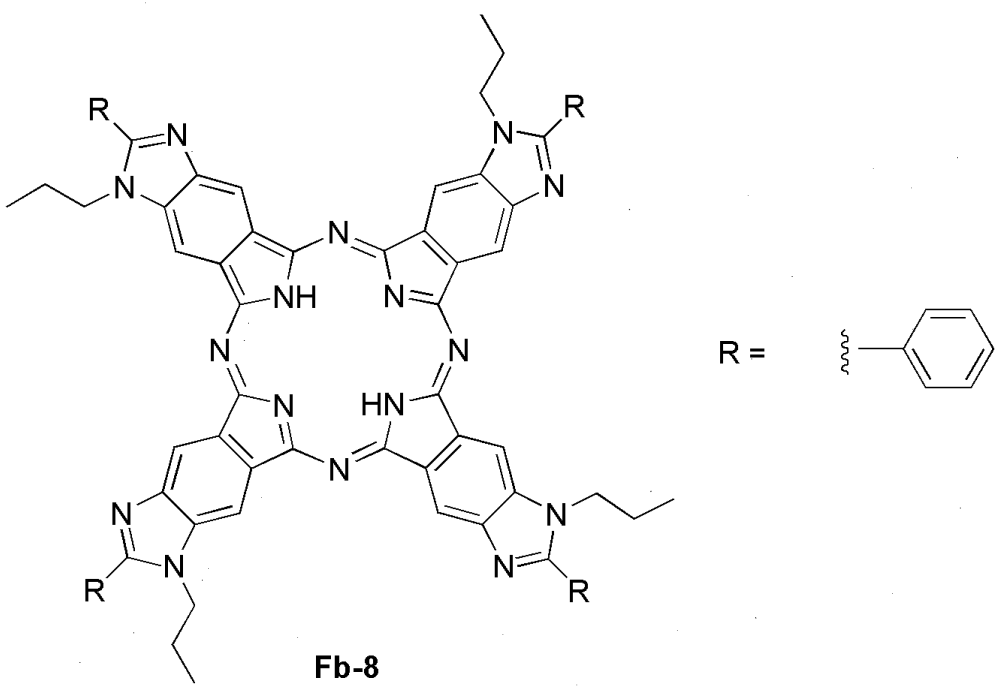




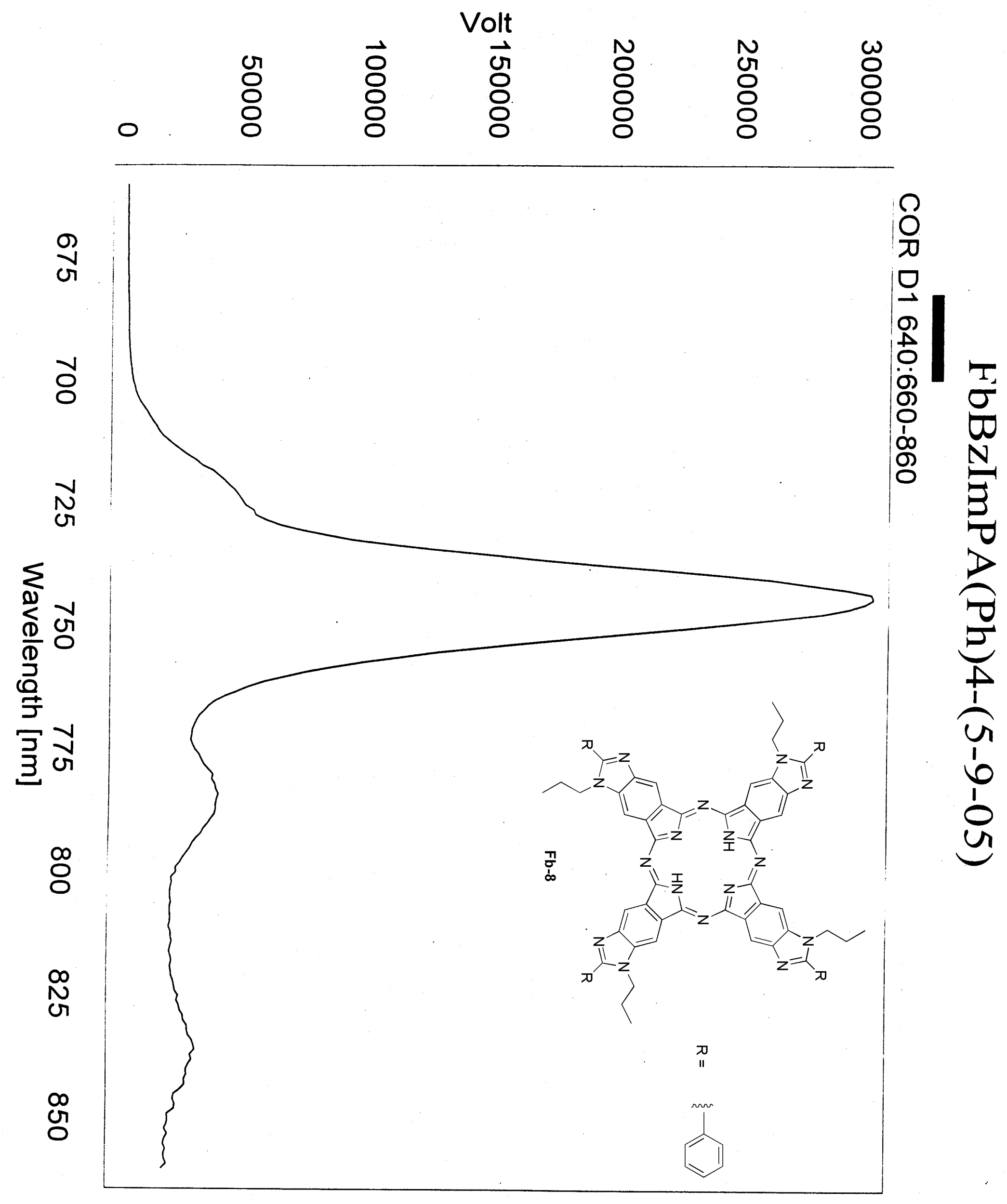




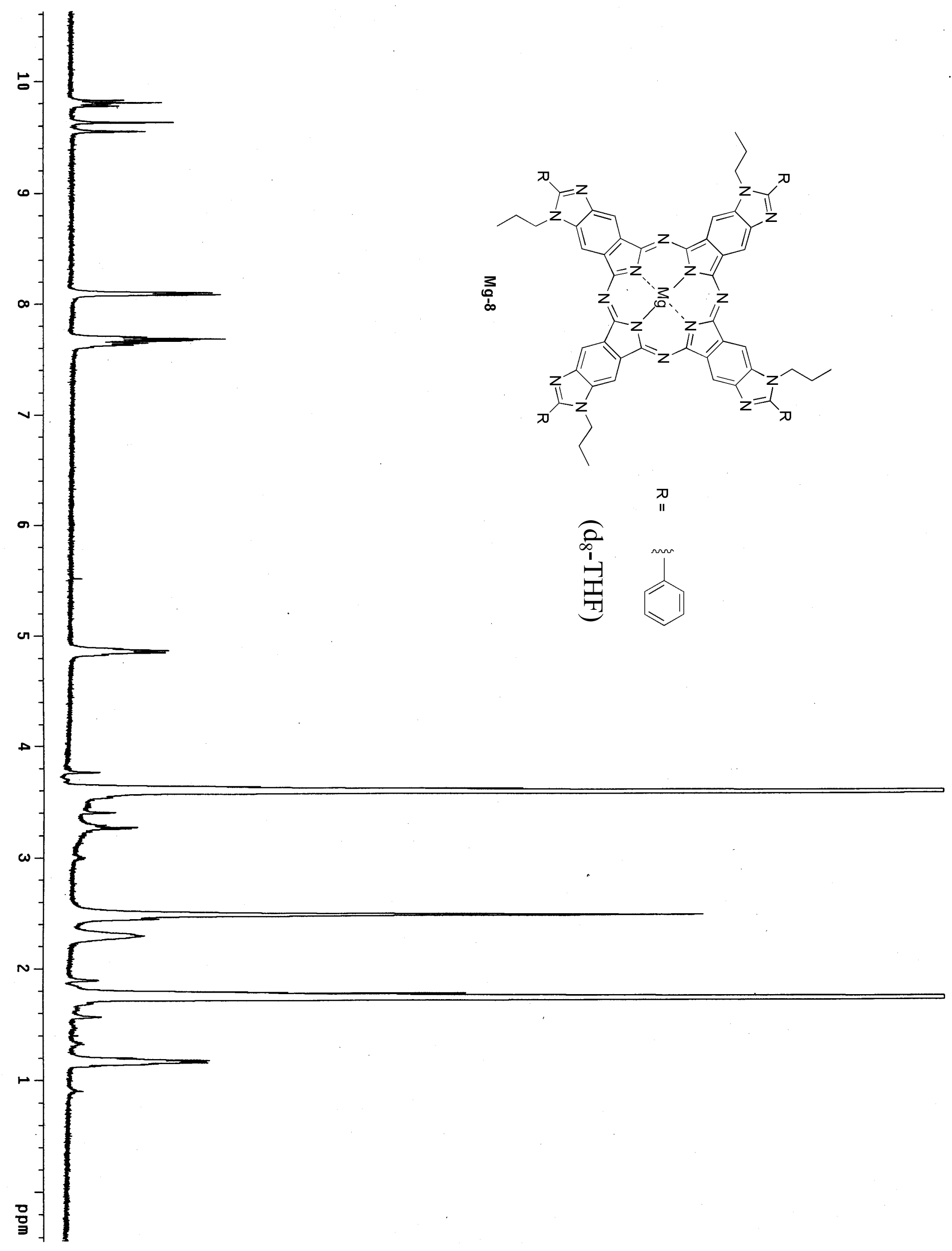




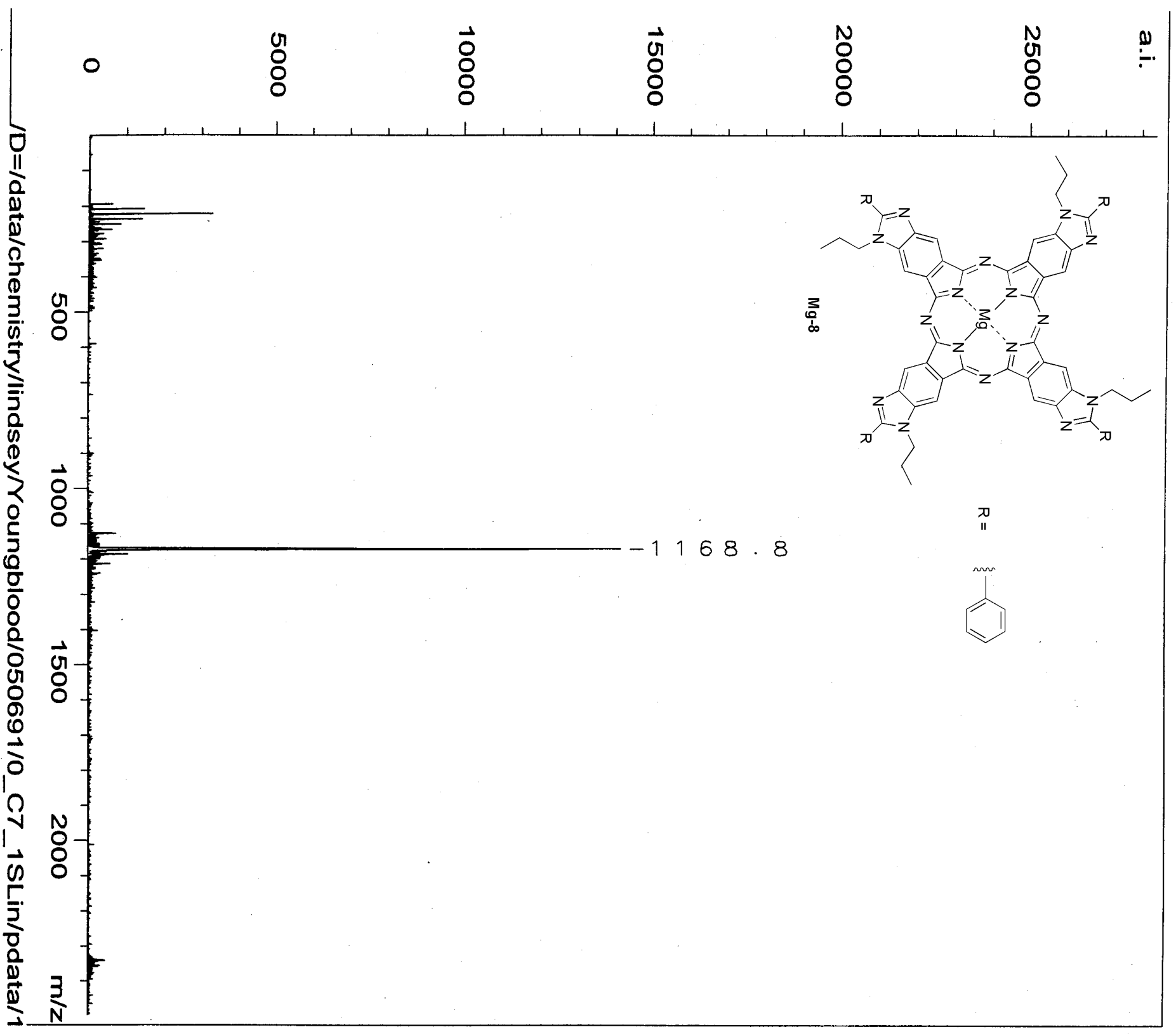

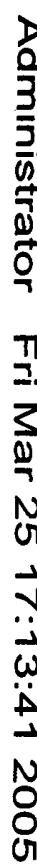

คด̄ उ3N

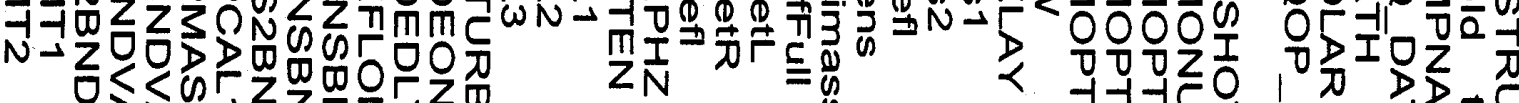

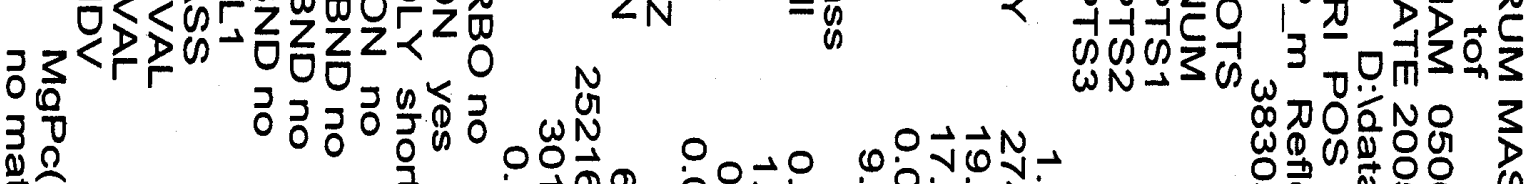

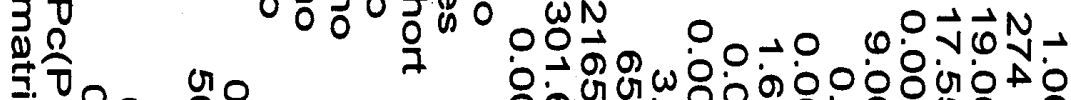
它. 的凹O

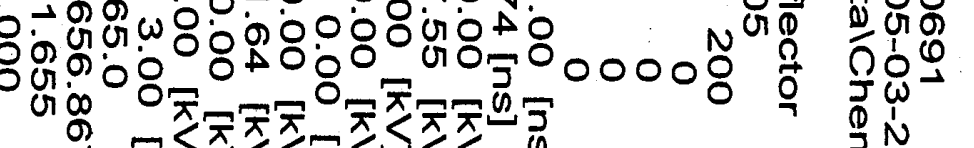

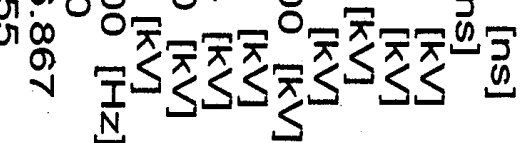

Ð 

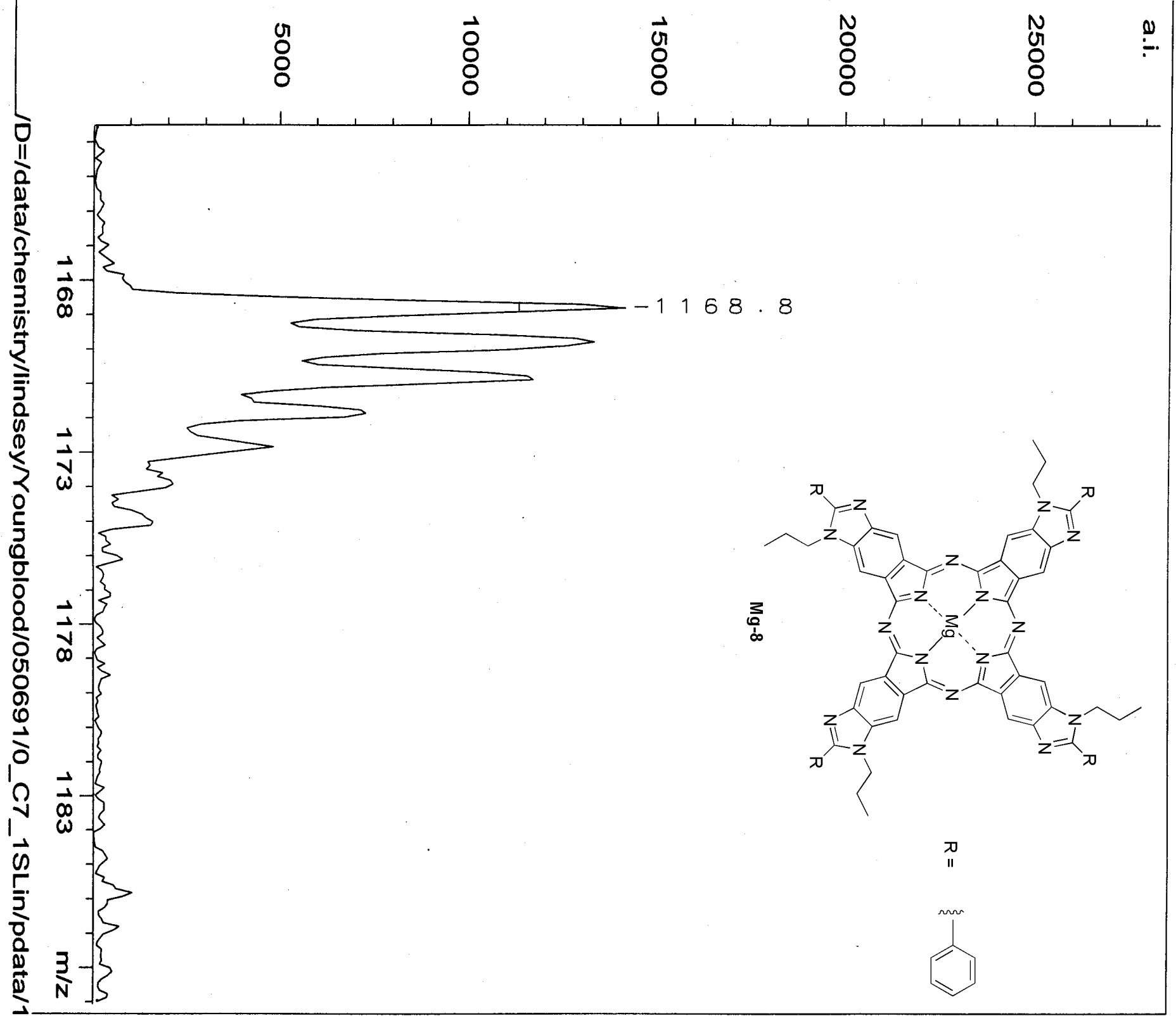

2
3
3
0
3
0
0
0
0
$\vdots$
0
0
0
$N$
0
0

ڤด̄ $33 N z$ mo

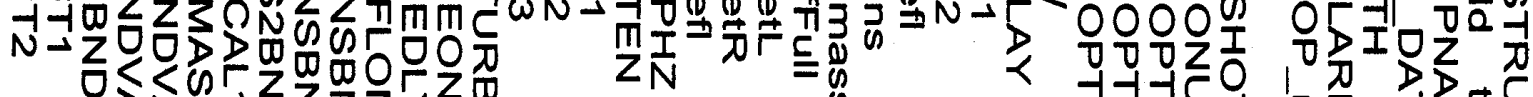

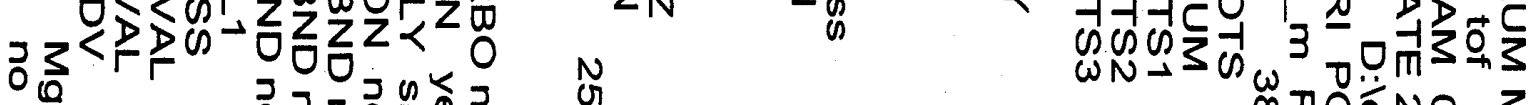

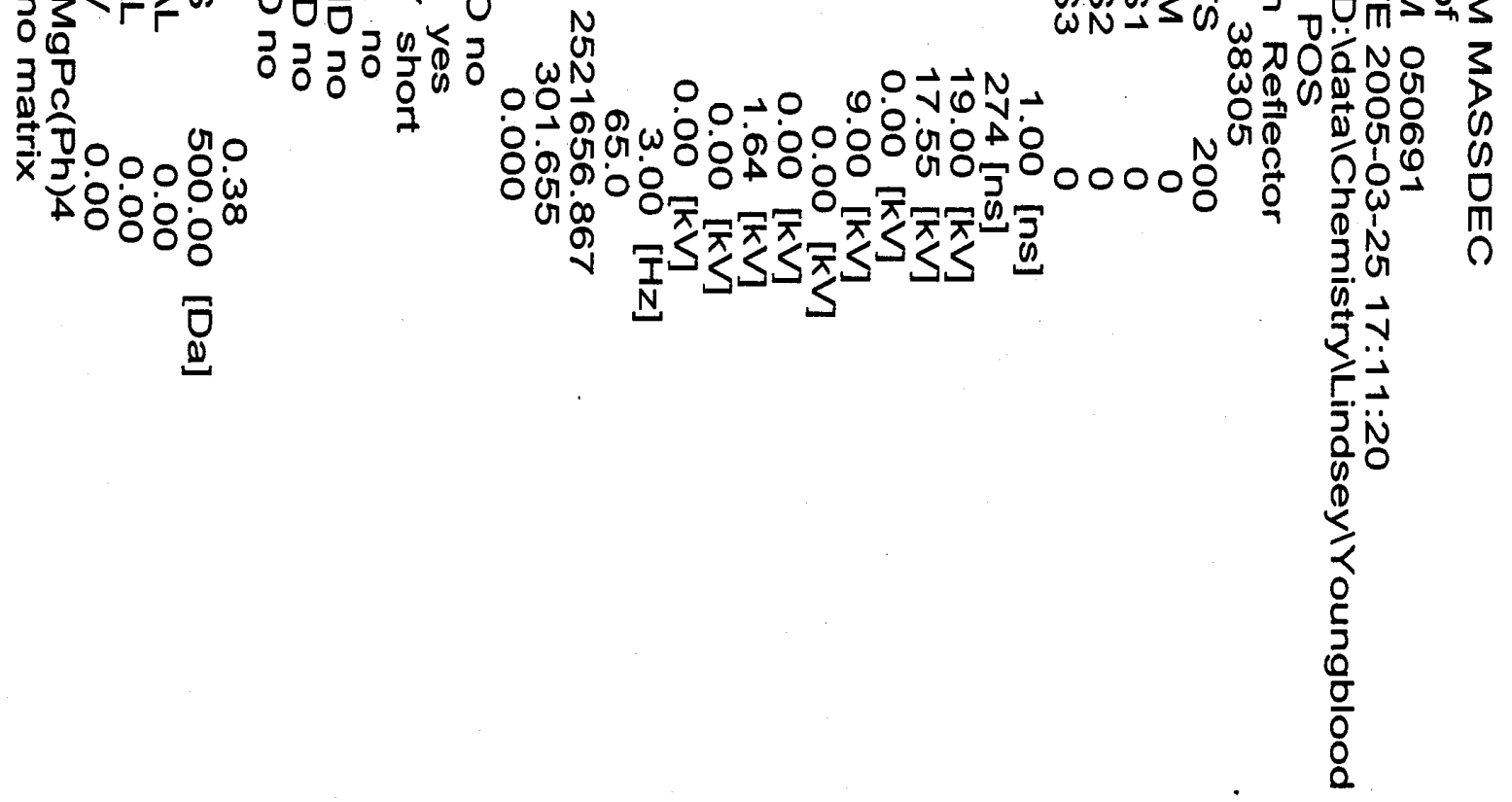


Method file: Information : Data File : <untitled>

Default Method

C: \UUSTIN \5-9-05 \MGPH 4 PA. SD

$17: 31: 09$

Overlaid Spectra:

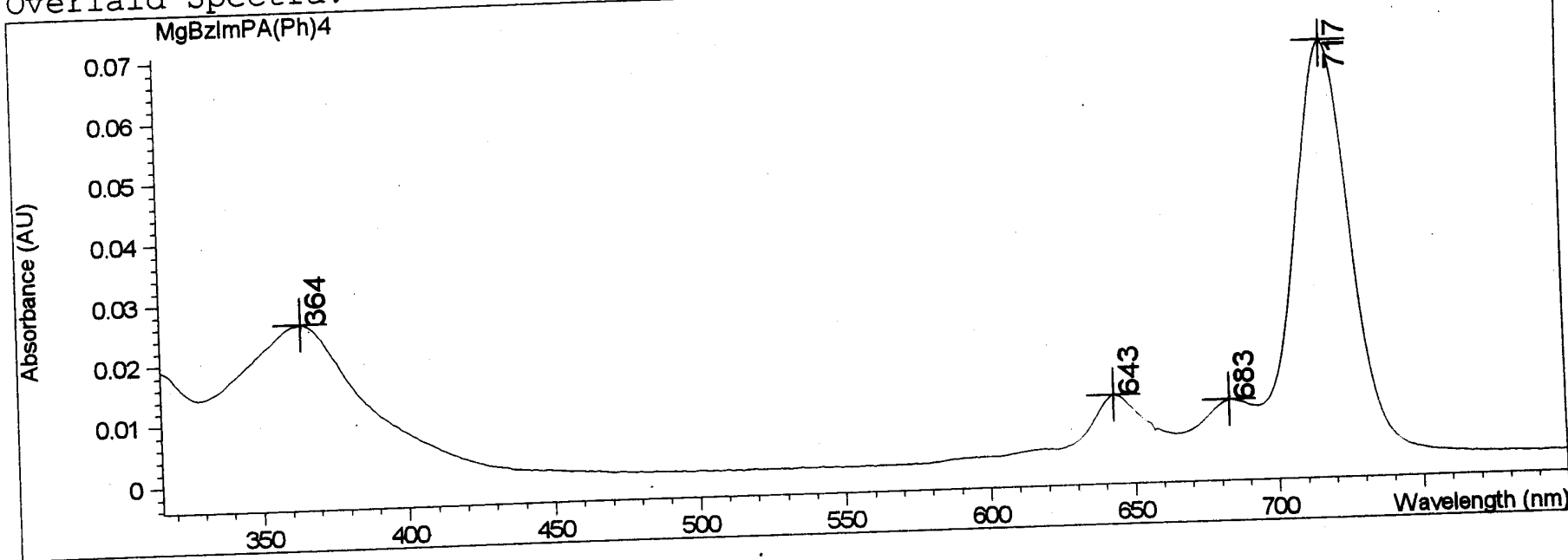

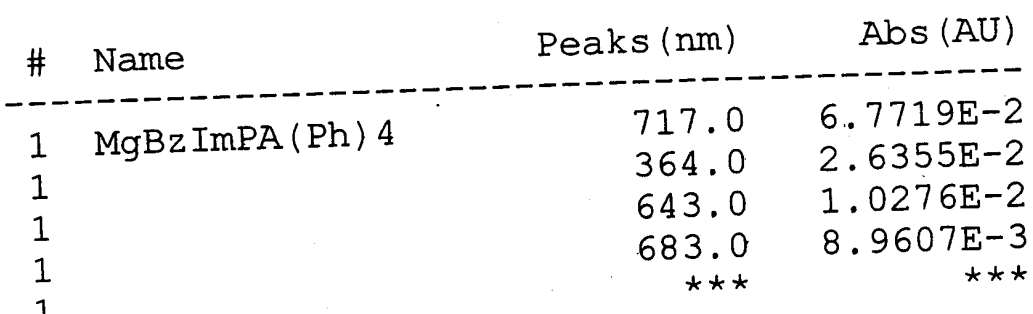

Report generated by :

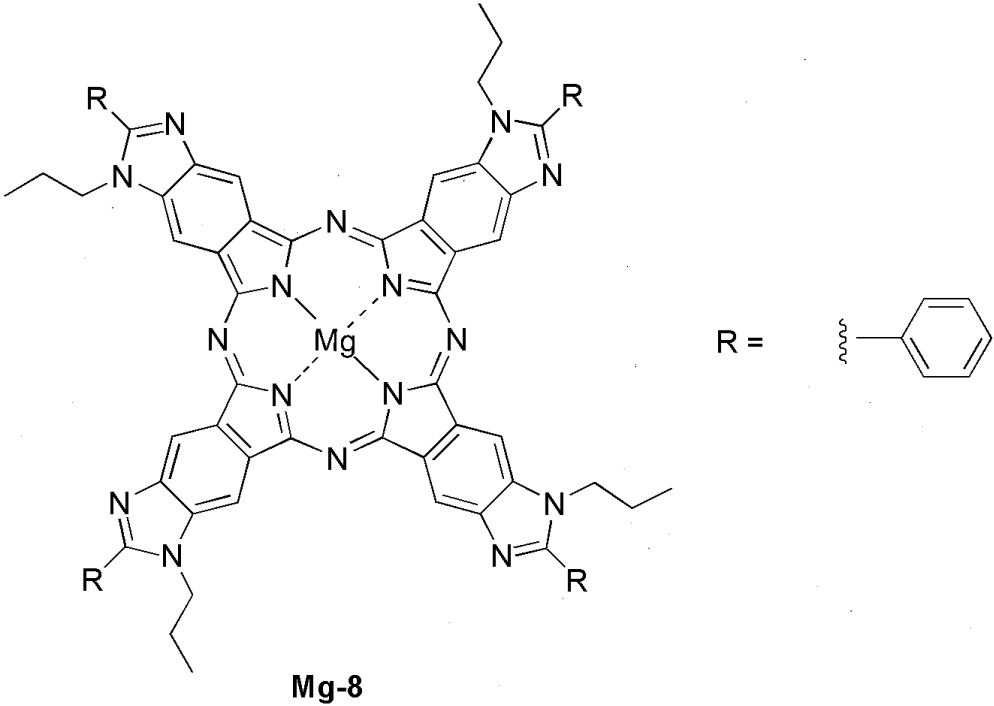




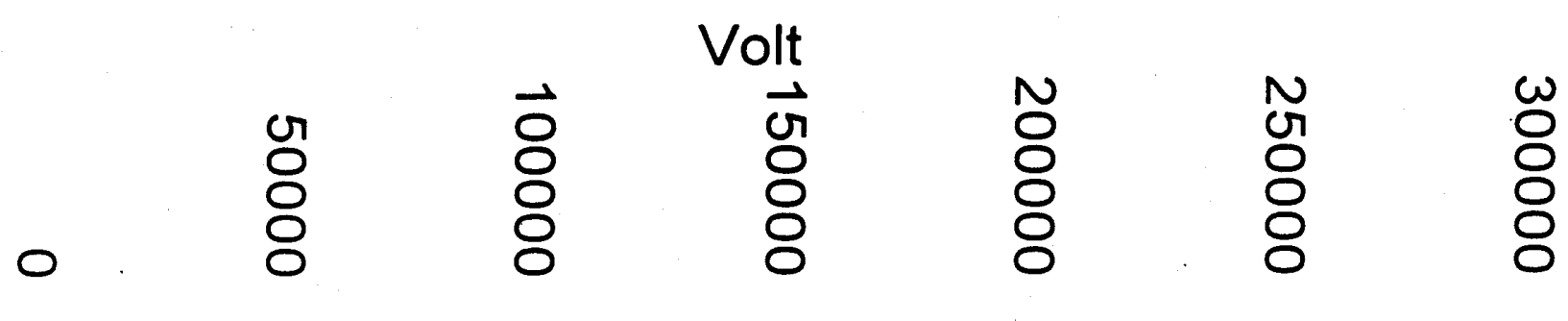

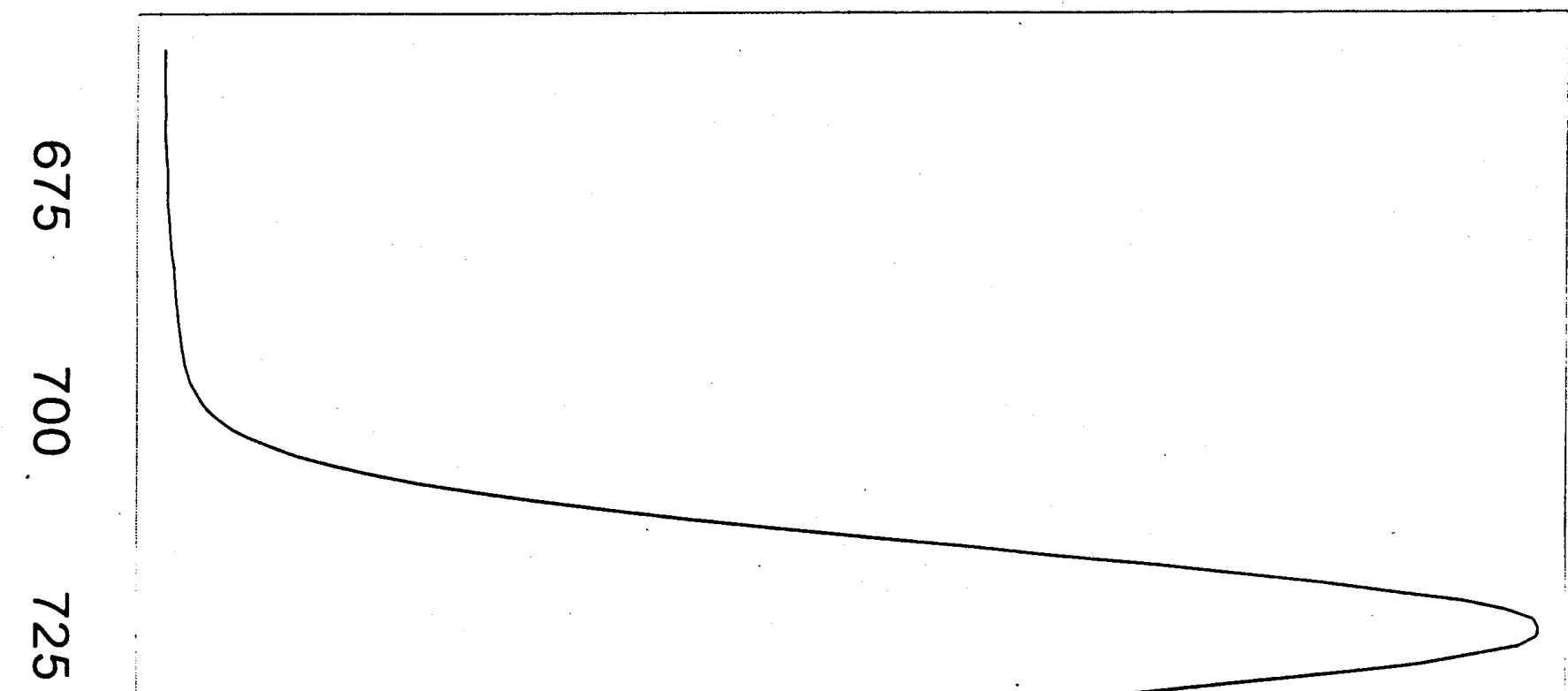

8

○

N
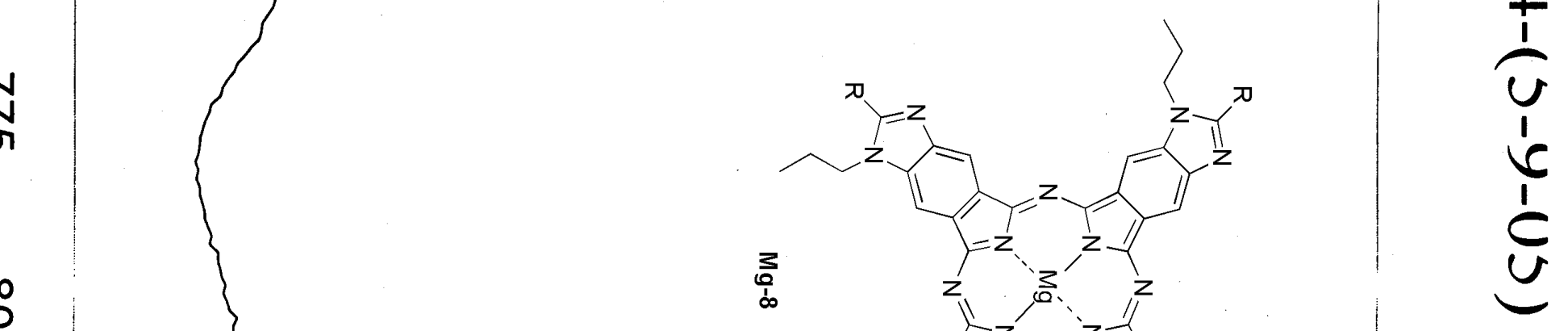


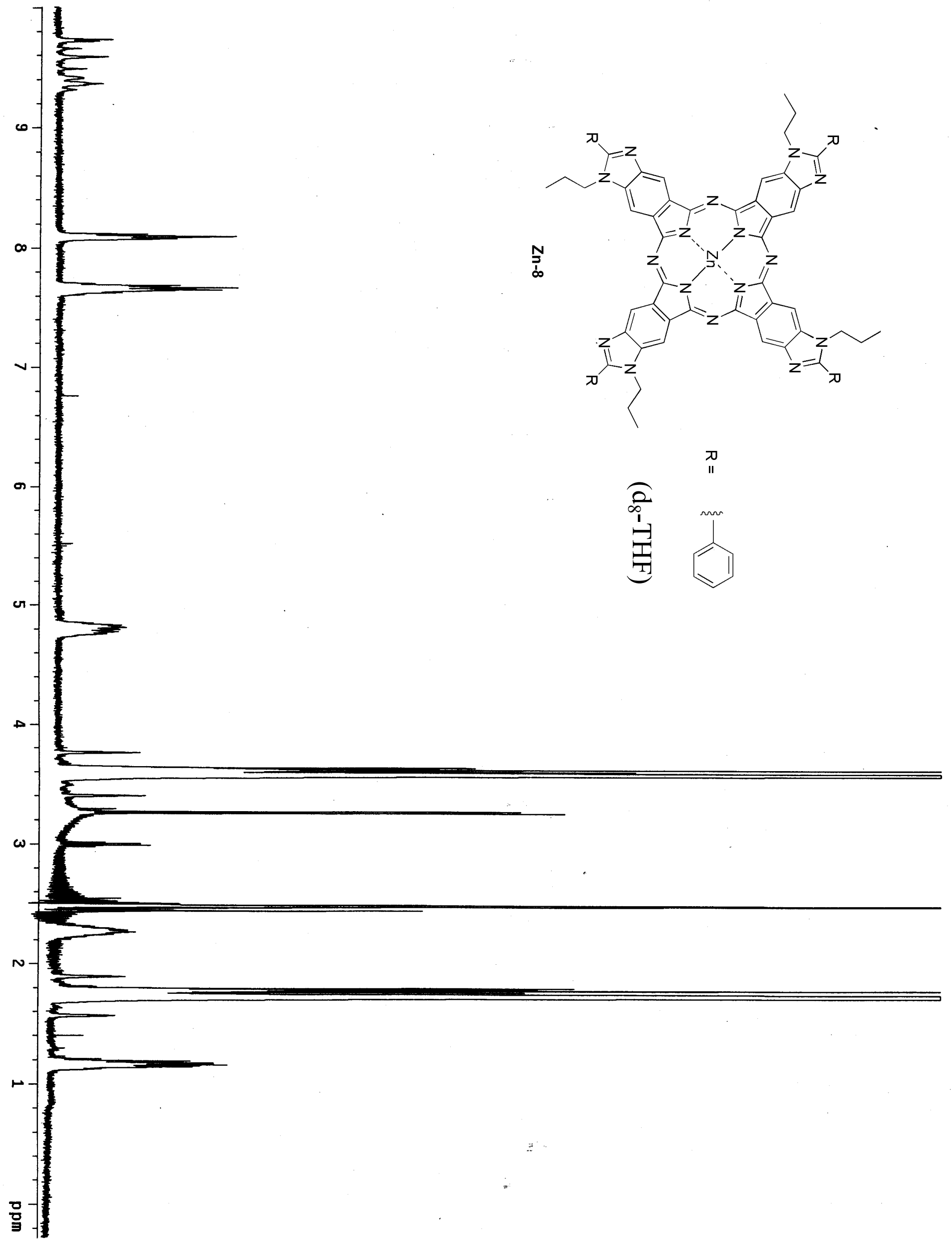

S 42 


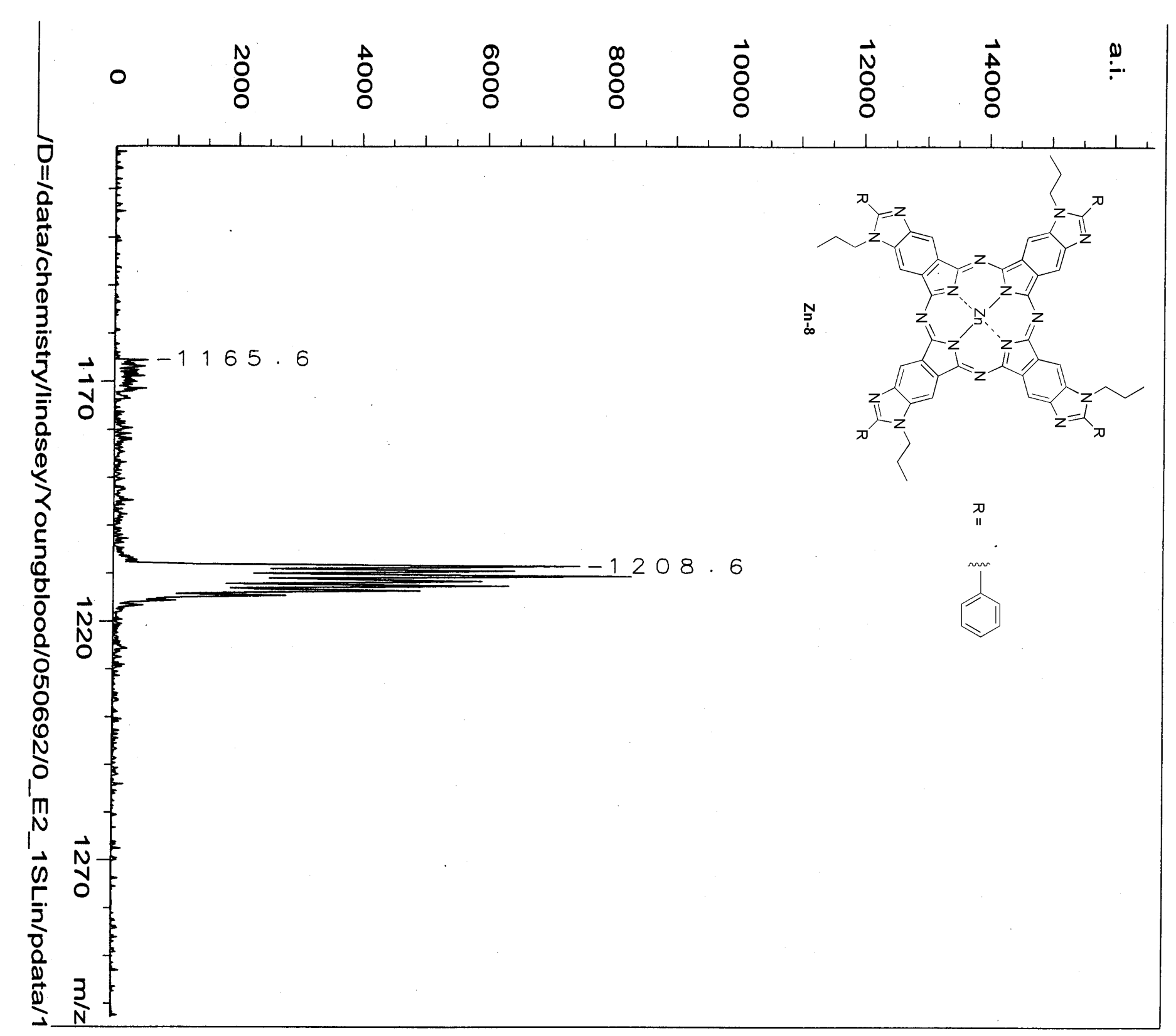

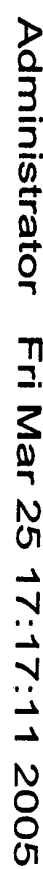

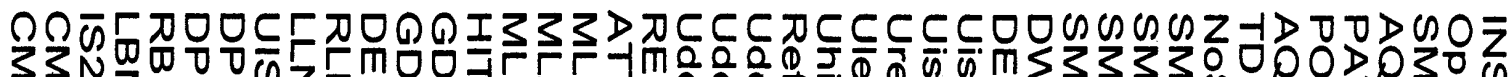

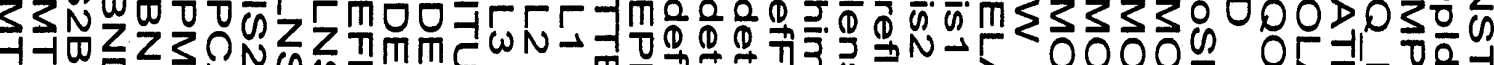

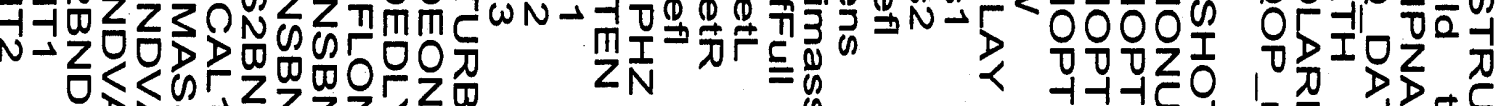

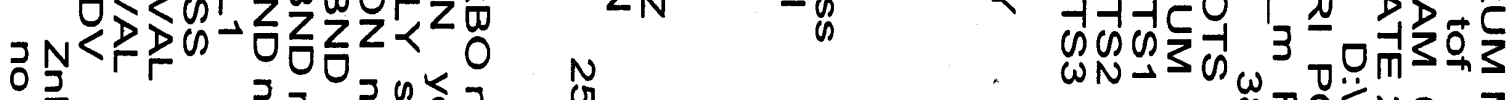

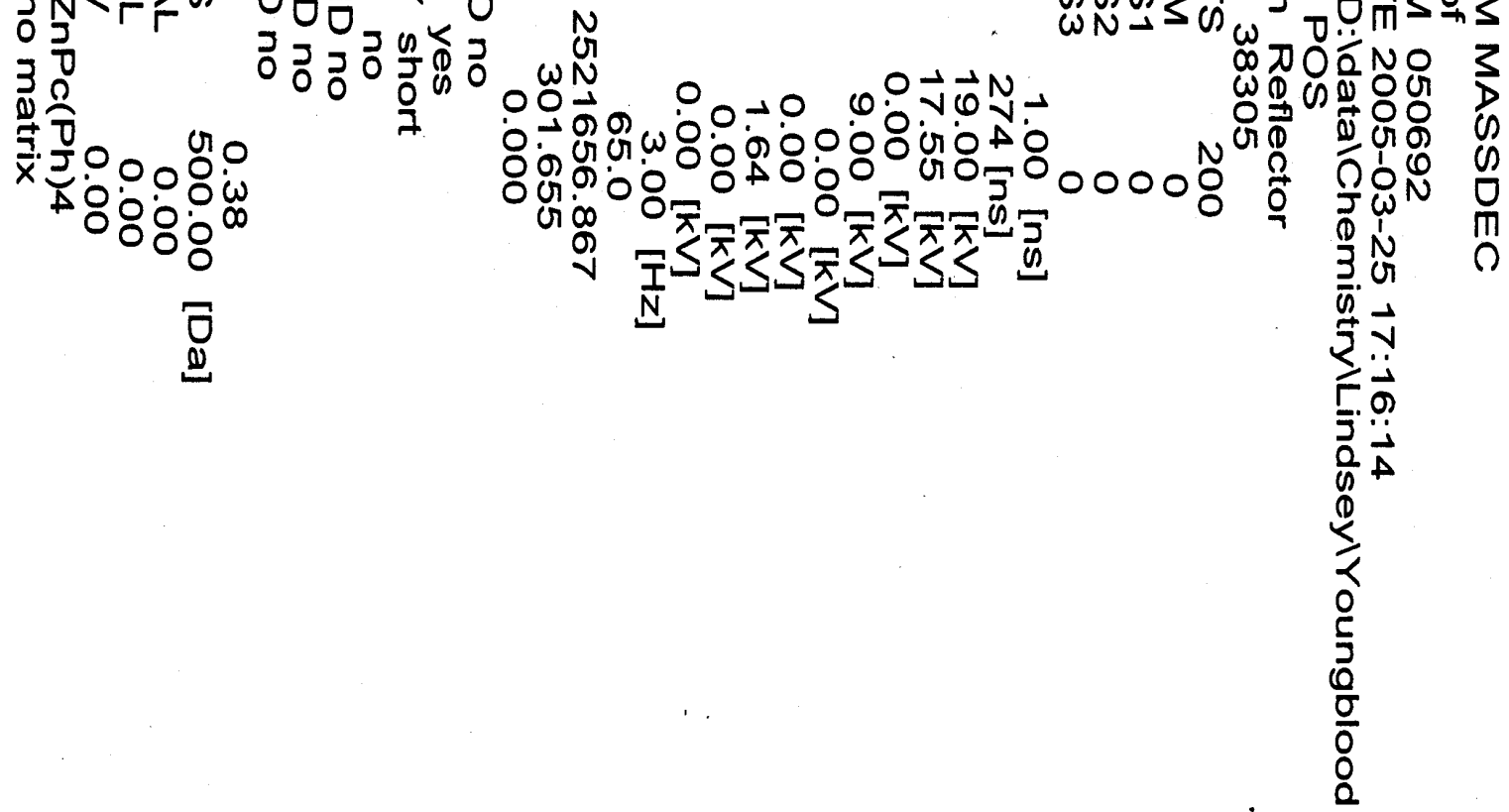


Method file : Information : Data File : <untitled>

Default Method

C: \UUSTIN \5-9-05\ZNPH4PA.SD Created : 5/9/05

$16: 07: 36$

Overlaid Spectra:

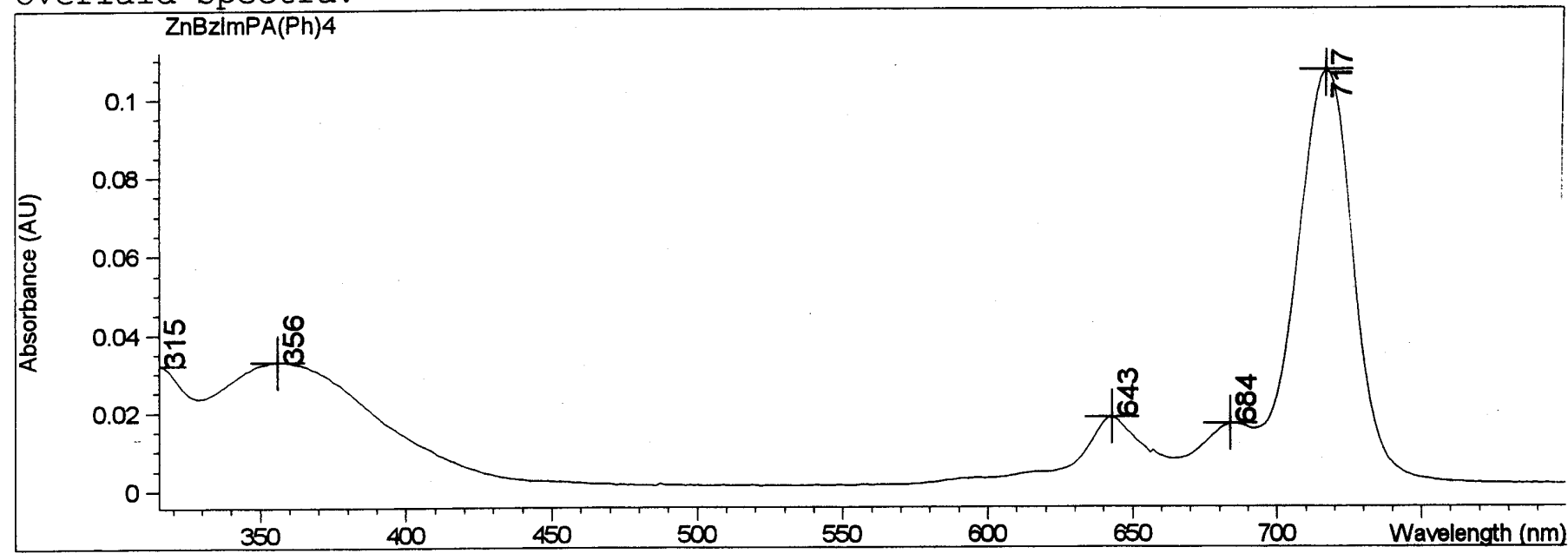

\begin{tabular}{lrrr}
$\#$ & Name & Peaks $(\mathrm{nm})$ & Abs (AU) \\
\hline 1 & $\operatorname{ZnBzImPA}(\mathrm{Ph}) 4$ & 717.0 & 0.10705 \\
1 & & 356.0 & $3.3036 \mathrm{E}-2$ \\
1 & 315.0 & $3.2072 \mathrm{E}-2$ \\
1 & 643.0 & $1.8526 \mathrm{E}-2$ \\
1 & 684.0 & $1.6791 \mathrm{E}-2$
\end{tabular}

Report generated by :

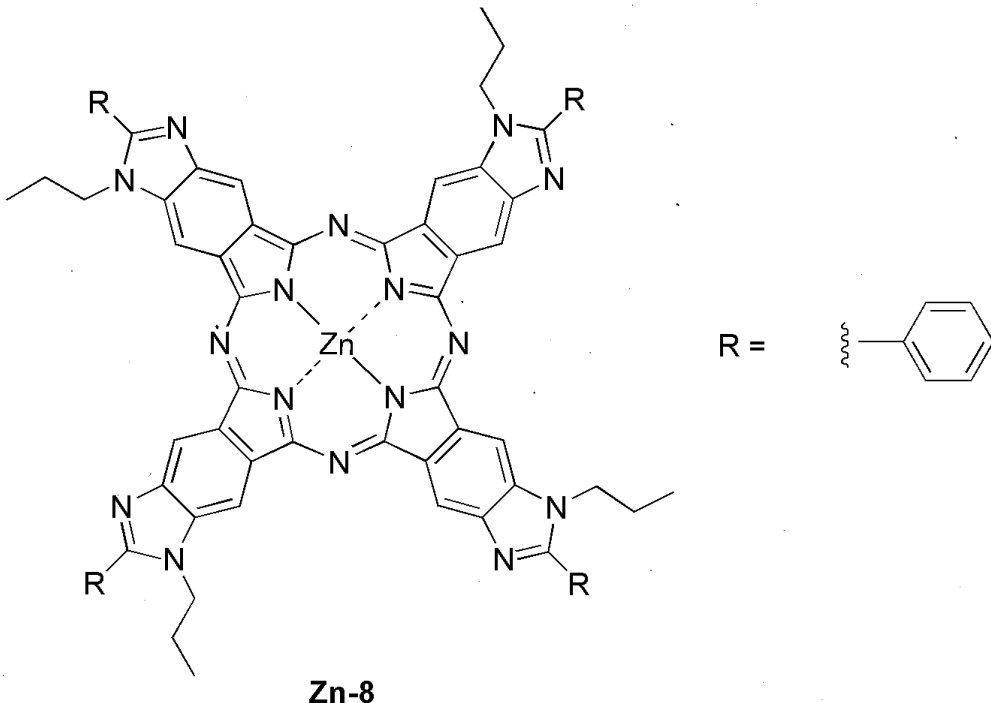




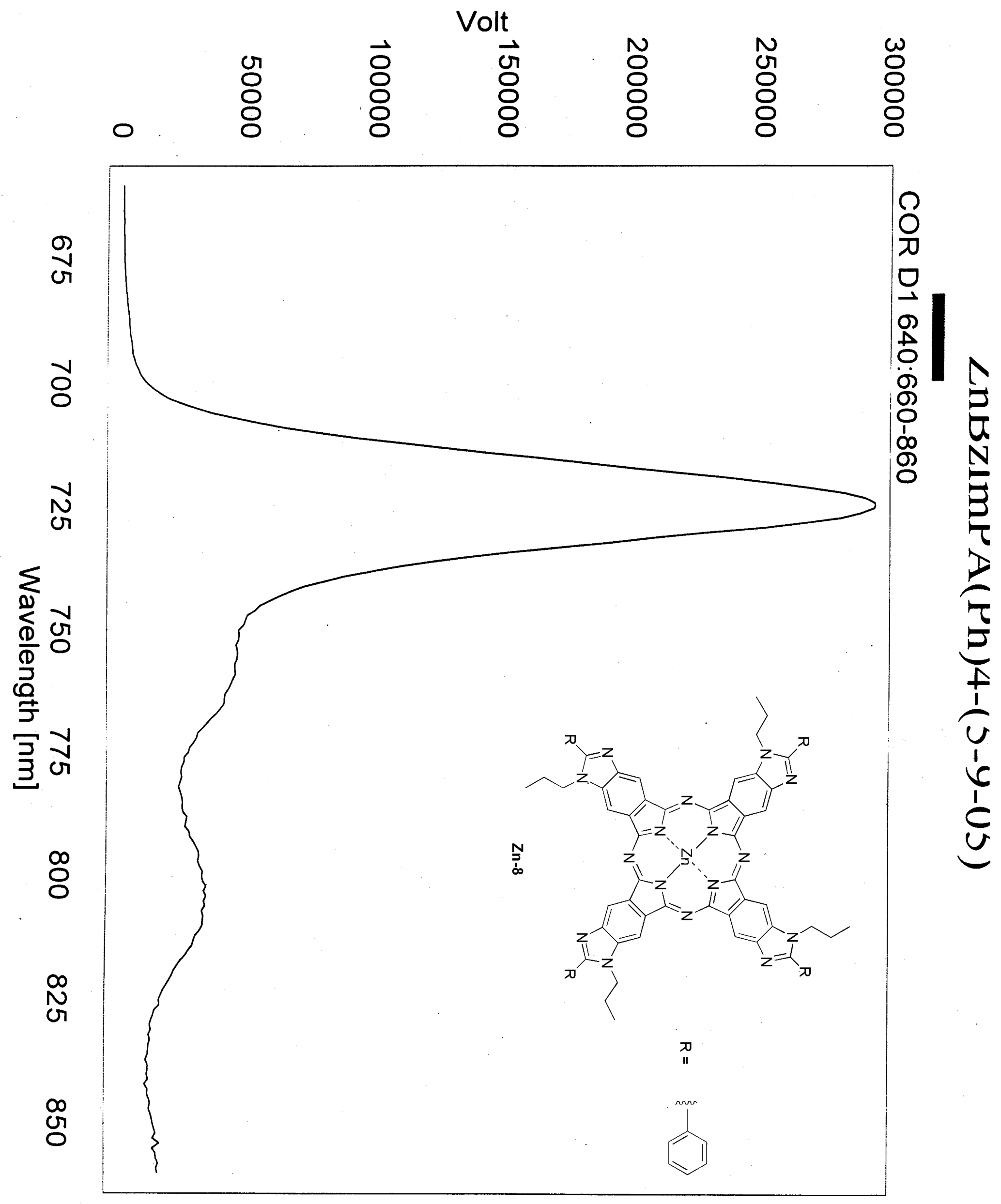




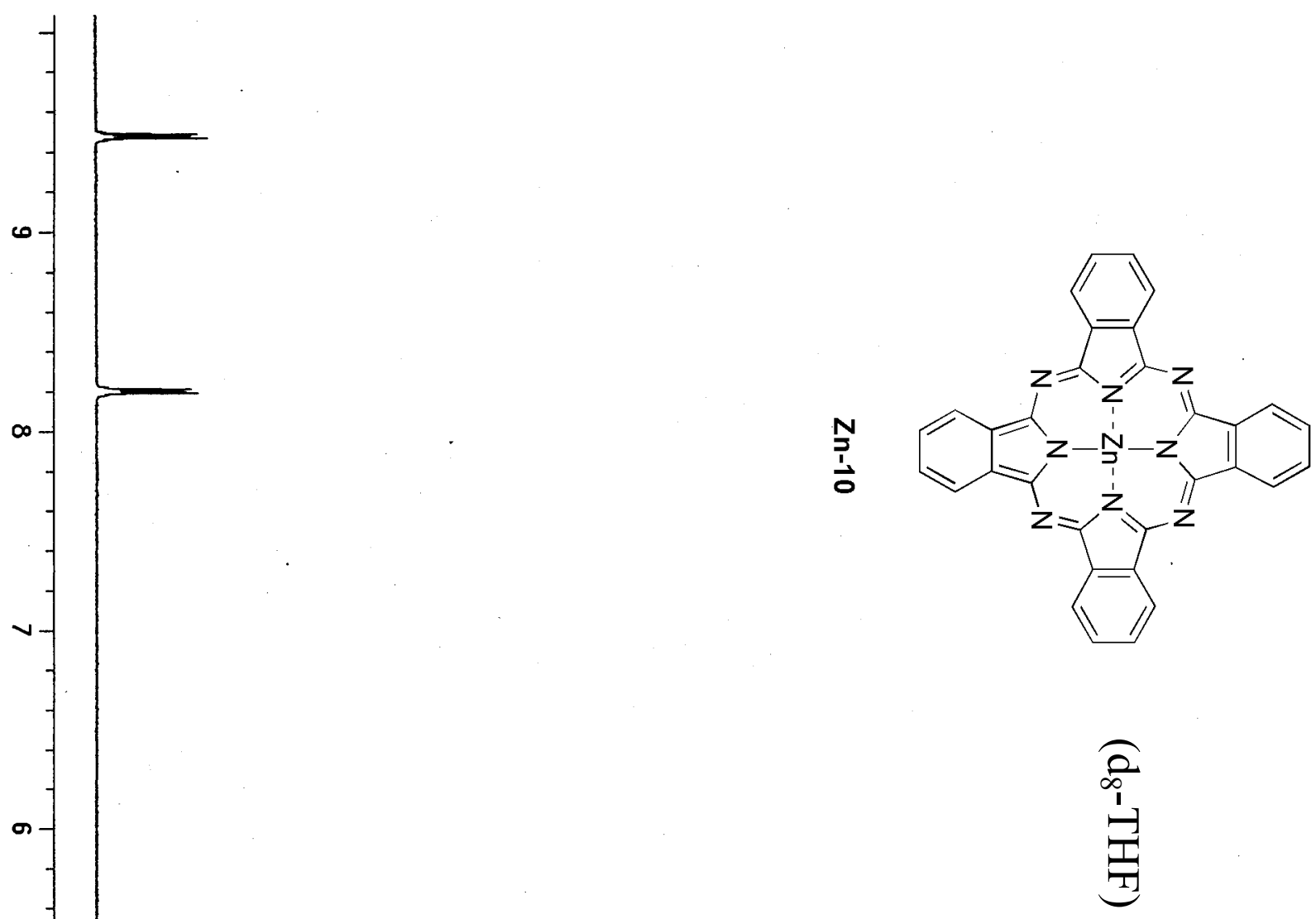

v

$\triangle$

$\varepsilon$

E-

S 47 


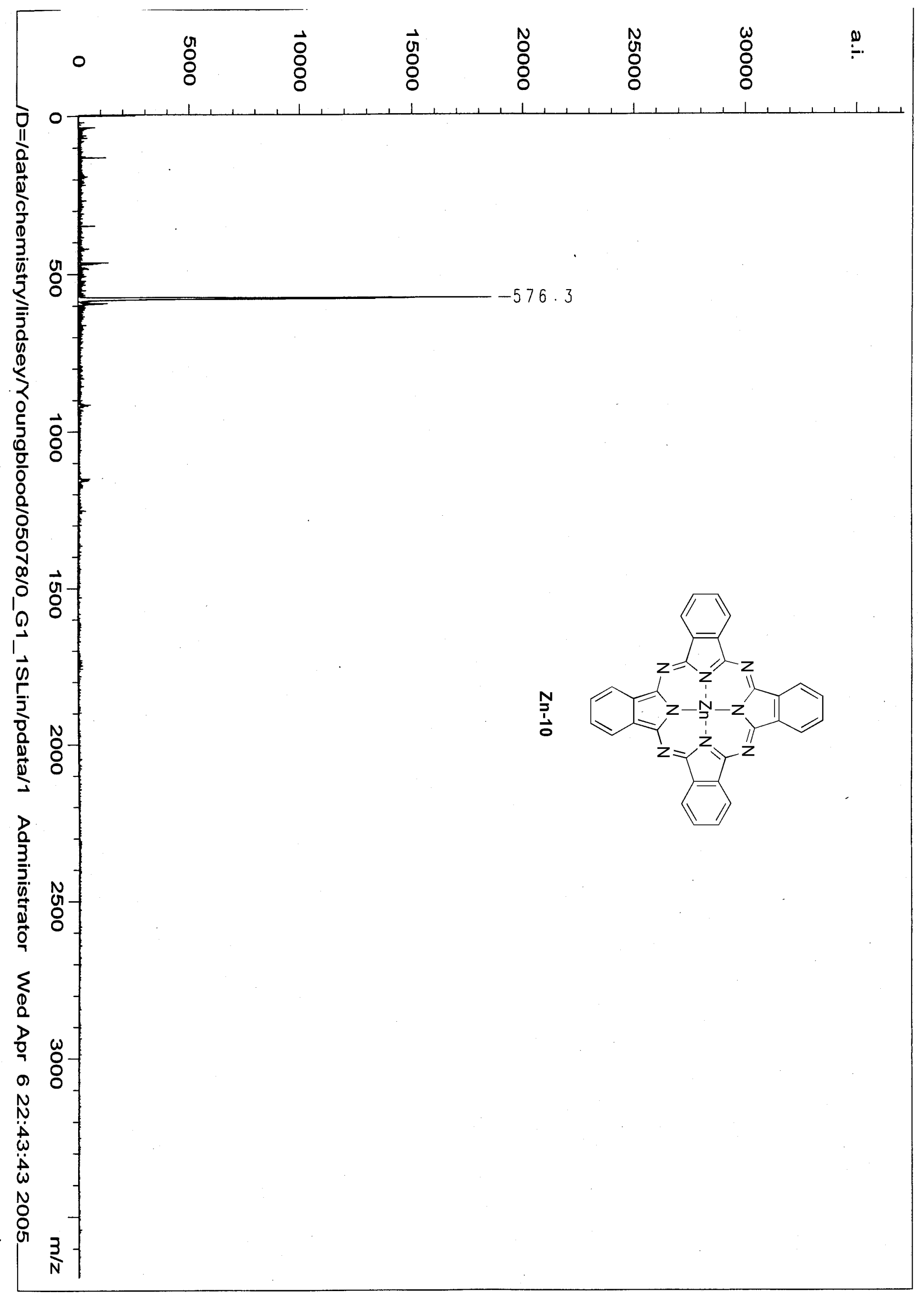




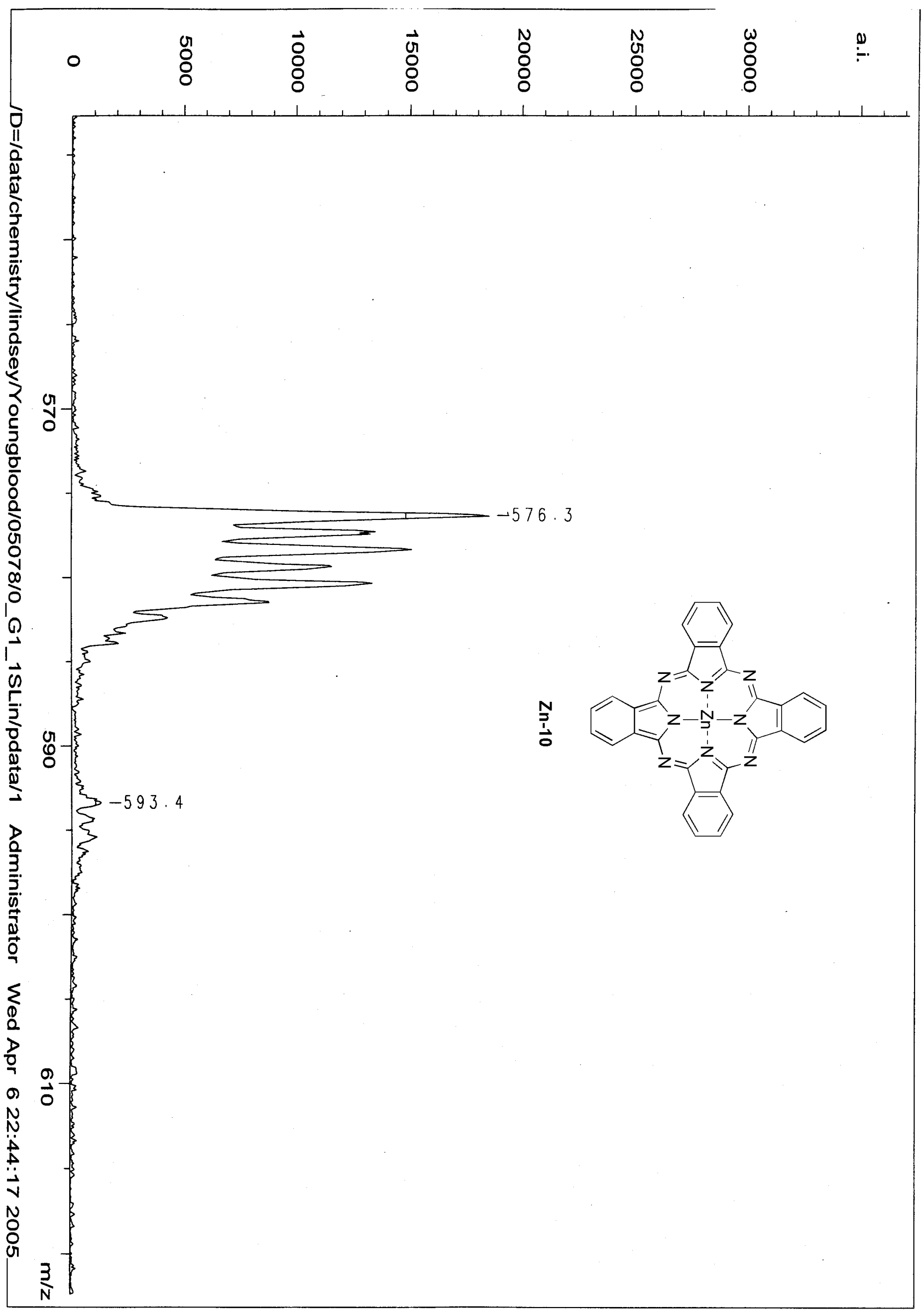


Method file : Information : Data File : <untitled>

Default Method

<untitled>

Overlaid Spectra:

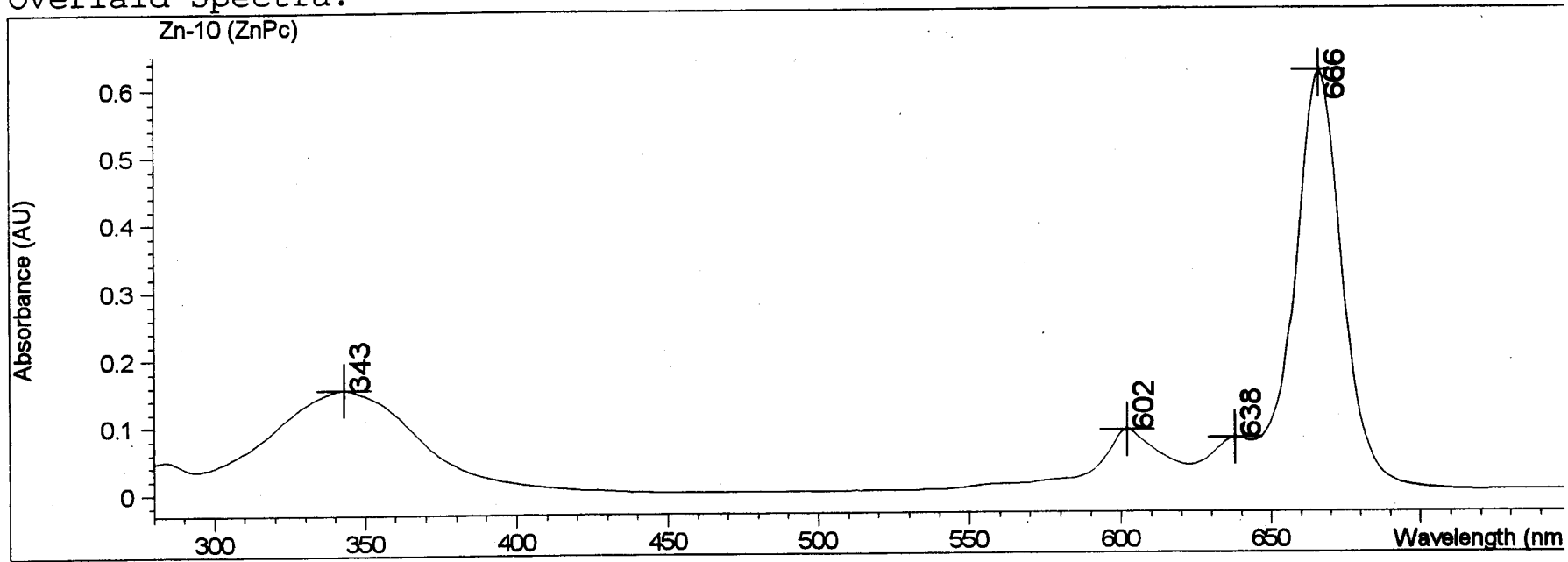

\begin{tabular}{|c|c|c|c|}
\hline \# & Name & Peaks (nm) & $\operatorname{Abs}(A U)$ \\
\hline 1 & $2 n-10$ & 666.0 & 0.62101 \\
\hline 1 & & 343.0 & 0.15569 \\
\hline 1 & & 602.0 & $8.9423 \mathrm{E}-2$ \\
\hline 1 & & 638.0 & $7.7984 \mathrm{E}-2$ \\
\hline
\end{tabular}

Report generated by :

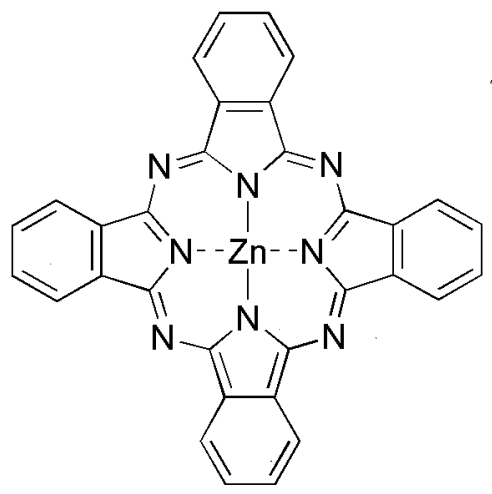

$\mathrm{Zn}-10$ 